\title{
The CALIFA view on stellar angular momentum across the Hubble sequence
}

\author{
J. Falcón-Barroso ${ }^{1,2}$, G. van de $\operatorname{Ven}^{3}$, M. Lyubenova ${ }^{4}$, J. Mendez-Abreu ${ }^{1,2}$, J. A. L. Aguerri ${ }^{1,2}$, B. García-Lorenzo ${ }^{1,2}$, \\ S. Bekeraité ${ }^{5}$, S. F. Sánchez ${ }^{6}$, B. Husemann ${ }^{7}$, R. García-Benito ${ }^{8}$, R. M. González Delgado ${ }^{8}$, D. Mast ${ }^{9,10}$, \\ C. J. Walcher ${ }^{5}$, S. Zibetti ${ }^{11}$, L. Zhu ${ }^{12}$, J. K. Barrera-Ballesteros ${ }^{6}$, L. Galbany ${ }^{13}$, P. Sánchez-Blázquez ${ }^{14}$, R. Singh ${ }^{7}$, \\ R. C. E. van den Bosch ${ }^{7}$, V. Wild ${ }^{15}$, J. Bland-Hawthorn ${ }^{16,17}$, R. Cid Fernandes ${ }^{18}$, A. de Lorenzo-Cáceres ${ }^{1,2}$, \\ A. Gallazzi ${ }^{11}$, R. A. Marino ${ }^{19}$, I. Márquez ${ }^{8}$, R. F. Peletier ${ }^{20}$, E. Pérez ${ }^{8}$, I. Pérez ${ }^{21,22}$, M. M. Roth ${ }^{5}$, \\ F. F. Rosales-Ortega ${ }^{23}$, T. Ruiz-Lara ${ }^{1,2}$, L. Wisotzki ${ }^{5}$, and B. Ziegler ${ }^{3}$
}

(Affiliations can be found after the references)

Received 30 July 2019 / Accepted 11 October 2019

\begin{abstract}
We present the apparent stellar angular momentum over the optical extent of 300 galaxies across the Hubble sequence using integral-field spectroscopic (IFS) data from the CALIFA survey. Adopting the same $\lambda_{\mathrm{R}}$ parameter previously used to distinguish between slow and fast rotating early-type (elliptical and lenticular) galaxies, we show that spiral galaxies are almost all fast rotators, as expected. Given the extent of our data, we provide relations for $\lambda_{\mathrm{R}}$ measured in different apertures (e.g. fractions of the effective radius: $0.5 R_{\mathrm{e}}, R_{\mathrm{e}}, 2 R_{\mathrm{e}}$ ), including conversions to long-slit $1 \mathrm{D}$ apertures. Our sample displays a wide range of $\lambda_{\mathrm{Re}}$ values, consistent with previous IFS studies. The fastest rotators are dominated by relatively massive and highly star-forming Sb galaxies, which preferentially reside in the main star-forming sequence. These galaxies reach $\lambda_{\mathrm{Re}}$ values of $\sim 0.85$, and they are the largest galaxies at a given mass, while also displaying some of the strongest stellar population gradients. Compared to the population of S0 galaxies, our findings suggest that fading may not be the dominant mechanism transforming spirals into lenticulars. Interestingly, we find that $\lambda_{\mathrm{Re}}$ decreases for late-type Sc and Sd spiral galaxies, with values that occasionally set them in the slow-rotator regime. While for some of them this can be explained by their irregular morphologies and/or face-on configurations, others are edge-on systems with no signs of significant dust obscuration. The latter are typically at the low-mass end, but this does not explain their location in the classical $(V / \sigma, \varepsilon)$ and $\left(\lambda_{\text {Re }}, \varepsilon\right)$ diagrams. Our initial investigations, based on dynamical models, suggest that these are dynamically hot disks, probably influenced by the observed important fraction of dark matter within $R_{\mathrm{e}}$.
\end{abstract}

Key words. galaxies: kinematics and dynamics - galaxies: elliptical and lenticular, cD - galaxies: spiral - galaxies: structure galaxies: evolution - galaxies: formation

\section{Introduction}

After mass, one of the key parameters determining the fate of a galaxy is its angular momentum. A robust result from cosmological simulations is that the angular momentum distribution of dark matter halos is nearly constant with redshift (e.g. Bullock et al. 2001). The amount of angular momentum that is being transferred to the baryons is then believed to set the size of galactic disks (Mo et al. 1998) and to form the basis for the mass-size relation of galaxies (Shen et al. 2003). At the same time, tidal interactions and in particular mergers between galaxies can disturb or even fully destroy the disk so that the memory of the initial angular momentum might well be lost (e.g. Toomre \& Toomre 1972).

Galaxy mergers are indeed believed to be an important reason why spheroid-dominated galaxies with surface brightness profiles close to de Vaucouleur (with a Sérsic index $n \sim 4$ ) deviate from the mass-size relation of galaxies with outer surface brightness profiles close to exponential $(n \sim 1)$. The latter include disk-dominated spiral galaxies, but the relation seems to extend toward lower masses, including dwarf elliptical galaxies (e.g. Kormendy \& Bender 2012) and possibly even down to the dwarf spheroidal galaxies (e.g. Brasseur et al. 2011).

Even though stellar rotation is observed in dwarf elliptical galaxies (e.g. Toloba et al. 2011) and possibly even in dwarf spheroidal galaxies (e.g. Battaglia et al. 2008), the motion of their stars remains dominated by dispersion. This implies that the initial angular momentum that set their sizes had been reduced, likely as a result of the mechanisms that are believed to have transformed dwarf disk galaxies into these dwarf spheroid galaxies. Transformation mechanisms which have been proposed, such as tidal interaction and ram pressure stripping, are thought to act stochastically, as reflected in the large diversity in photometric, kinematic and stellar population properties (e.g. Ryś et al. 2013, 2014, 2015), but sudden dramatic changes as a result of, for example, mergers are expected to be rare (Amorisco et al. 2014).

This shows that even if the process of transferring the angular momentum from halo to disk is broadly understood (e.g. Burkert et al. 2016), there still might not be a direct link between the size of the disk of galaxy and its current stellar angular momentum. However, the comparison between current angular momentum and size of galaxies at a given mass provides constraints on the changes in angular momentum and on the mechanisms that caused these changes. The latter mechanisms, in turn, are in all likelihood the same that are responsible for defining the Hubble sequence of galaxies (e.g. Romanowsky \& Fall 2012). Clearly, a crucial ingredient in uncovering the evolution of galaxies is a homogeneous and statistically-sound census of the stellar angular momentum in nearby galaxies of all Hubble types.

The SAURON project (de Zeeuw et al. 2002) combines the observed stellar line-of-sight velocity and dispersion fields of 
48 early-type galaxies to compute the parameter $\lambda_{\mathrm{Re}}$ as a measure of the apparent stellar angular momentum within one effective radius $R_{\mathrm{e}}$ (Emsellem et al. 2007). The ATLAS ${ }^{3 \mathrm{D}}$ survey (Cappellari et al. 2011a) extended this to a volume-limited sample of 260 early-type galaxies out to $42 \mathrm{Mpc}$ to confirm the existence of two families: slow rotators elliptical galaxies with complex stellar velocity fields and fast rotator lenticular as well as elliptical galaxies with regular stellar velocity fields (Krajnović et al. 2011). With the advent of new 2D surveys (e.g. SAMI, Croom et al. 2012; SLUGGS, Arnold et al. 2014; MASSIVE, Ma et al. 2014; MaNGA, Bundy et al. 2015), there has been steady progress in this field over the past decade. While initial samples were still biased towards early-type systems (e.g. Arnold et al. 2014; Fogarty et al. 2014; Veale et al. 2017a), the topic has remained active and has spurred the study of angular momentum in even larger samples of galaxies (including spirals) by the SAMI (Cortese et al. 2016; hereafter C16; van de Sande et al. 2017; hereafter vdS17) and MaNGA (Graham et al. 2018; hereafter G18) survey teams.

The CALIFA survey (Sánchez et al. 2012) of a diameterselected sample of up to 600 nearby galaxies provides stellar velocity and dispersion fields that not only extend further out in radius, but also cover galaxies of all Hubble types. The goal of this paper is to use the stellar velocity and dispersion maps of 300 observed CALIFA galaxies presented in Falcón-Barroso et al. (2017; hereafter referred to as FLV17), in order to provide a robust census of the apparent stellar angular momentum across the Hubble sequence and investigate the properties of galaxies in some of the most extreme regions of the $\left(\lambda_{\mathrm{Re}}, \varepsilon\right)$ diagram. Part of the results shown here have already been presented in Falcón-Barroso et al. (2015), and have been used in recent literature for comparison with other surveys (e.g. Cappellari 2016; Schulze et al. 2018) or for highlighting the peculiarities of certain types of galaxies (e.g. del Moral-Castro et al. 2019).

The paper is organised as follows. After describing in Sect. 2 the available data for 300 galaxies, we present in Sect. 3 the resulting apparent stellar angular momentum within apertures of different radii and as radial profiles when only long-slit data is available. In Sect. 4 , we present the galaxies on the $(V / \sigma, \varepsilon)$ and $\left(\lambda_{\mathrm{Re}}, \varepsilon\right)$ diagrams to investigate the rotational versus pressure support homogeneously among galaxies of all morphologies, showing trends among types and discussing their relation to other global parameters. We conclude our findings in Sect. 5. Appendix A describes the procedures used to deproject our $\lambda_{\operatorname{Re}}$ measurements. Table B.1 provides all the quantities used and derived in our study. Throughout we adopt $H_{0}=$ $70 \mathrm{~km} \mathrm{~s}^{-1} \mathrm{Mpc}^{-1}, \Omega_{\mathrm{M}}=0.3$ and $\Omega_{\mathrm{L}}=0.7$ for respectively the Hubble constant, the matter density and the cosmological constant, although these parameters only have a small effect on the physical scales of the galaxies due to their relative proximity.

\section{CALIFA IFU and ancillary data}

The Calar Alto Legacy Integral Field Area (CALIFA) survey is the first integral-field spectroscopic (IFS) survey of a diameter selected $\left(45^{\prime \prime}<D_{25}<80^{\prime \prime}\right)$ sample of up to 600 galaxies in the local universe $(0.005<z<0.03)$ of all Hubble types (Sánchez et al. 2012). The so-called CALIFA "mother sample" of 938 galaxies, from which targets are randomly observed based on visibility, is representative in stellar mass over two orders of magnitude $9.4<\log \left(M_{\star} / M_{\odot}\right)<11.4$. This means that after a straightforward volume correction based on $D_{25}$, the mass (and corresponding luminosity) function over this range is recovered to better than $95 \%$ (Walcher et al. 2014). The $65^{\prime \prime} \times 72^{\prime \prime}$ field-of-view of the employed PMAS/PPAK-IFU (Kelz et al. 2006) covers the full optical extent of the selected galaxies, with a complete filling factor achieved through a three-point dithering scheme, and with a spatial sampling of $1^{\prime \prime}$ that over-samples the spatial resolution by about a factor three (Husemann et al. 2013). The typical Pont-Spread-Function size is FWHM 2.5" (Sánchez et al. 2016), that corresponds to an average physical resolution of $0.7 \mathrm{kpc}$ and a range between 0.2 and $1.5 \mathrm{kpc}$ within the considered redshift range.

In this study, we used the high-quality stellar kinematics presented in FLV17 from the V1200 dataset. Briefly, stellar velocity $(V)$ and velocity dispersion $(\sigma)$ maps were computed using the pPXF code of Cappellari \& Emsellem (2004), after the data had been Voronoi binned (Cappellari \& Copin 2003) to a signalto-noise ratio $(\mathrm{S} / \mathrm{N})$ of 20 per pixel. We use the Indo-US spectral library (Valdes et al. 2004) as templates over the wavelength range covered by the V1200 grating (i.e. $3750-4550 \AA$ ), which includes prominent absorption features such as $\mathrm{Ca} \mathrm{H}+\mathrm{K}, \mathrm{H} \gamma$ or the $\mathrm{H} \delta$ lines. The typical relative uncertainties of our measurements are $\sim 5 \%$ for $\sigma \geq 150 \mathrm{~km} \mathrm{~s}^{-1}$. Below that value they increase up to $50 \%$ for velocity dispersions as low as $20 \mathrm{~km} \mathrm{~s}^{-1}$. We refer the reader to FLV17 for more details.

Additional global galaxy properties used here are: (i) distances based on redshift with Hubble flow corrected for Virgo infall (see Walcher et al. 2014); (ii) SDSS redshifts, apparent magnitudes and corresponding colors; (iii) light concentrations based on SDSS $r$-band 50 and 90 percentile Petrosian radii; (iv) effective radii $\left(R_{\mathrm{e}}\right)$ estimated using a growth-curve analysis applied to the SDSS images as described in Walcher et al. (2014); (v) stellar masses based on Sunrise spectral energy distribution fits from Walcher et al. (2014); (vi) global star formation rates (SFRs) based on Balmer-decrement corrected $\mathrm{H} \alpha$ fluxes extracted from the CALIFA datacubes (Sánchez et al. 2017); and (vii) stellar population parameters (average ages and age gradients) from García-Benito et al. (2017) using CALIFA data Voronoi binned to reach a target $S / N \sim 20$. The resulting spectra of each bin was then processed using PyCASSO (Cid Fernandes et al. 2013; Amorín et al. 2017) using a combination of the GRANADA (González Delgado et al. 2005) and MILES models (Vazdekis et al. 2015) respectively. Reported ages are averages within $R_{\mathrm{e}}$, while radial age gradients are computed performing a robust linear fit over the entire inner $R_{\mathrm{e}}$.

\section{Apparent stellar angular momentum}

Following Emsellem et al. (2007), we defined the apparent stellar angular momentum as

$\lambda_{\mathrm{R}}=\frac{\sum_{j}^{N} F_{j} R_{j}\left|V_{j}\right|}{\sum_{j}^{N} F_{j} R_{j}\left(V_{j}^{2}+\sigma_{j}^{2}\right)^{\frac{1}{2}}}$

where $F_{j}, R_{j}, V_{j}$ and $\sigma_{j}$ are the flux, polar radius, velocity, and dispersion per spatial bin $j$ for which the centroid falls within an elliptic aperture with the given semi-major axis $R$, ellipticity $\varepsilon$, and position angle PA.

We adopted for $\varepsilon$ and PA the median values of the outer $10 \%$ radial points of, respectively, the ellipticity and position angle profile resulting from an IRAF ellipse model of the SDSS $r$-band image of each galaxy (Méndez-Abreu et al. 2017). This is in contrast to previous studies (e.g. Emsellem et al. 2007), where the mean ellipticity within $R_{\mathrm{e}}$ was used instead $\left(\varepsilon_{\mathrm{e}}\right)$. We decided on this option as the effect of non-axisymmetric distortions, which may be caused by, for example, bars, spiral arms, 


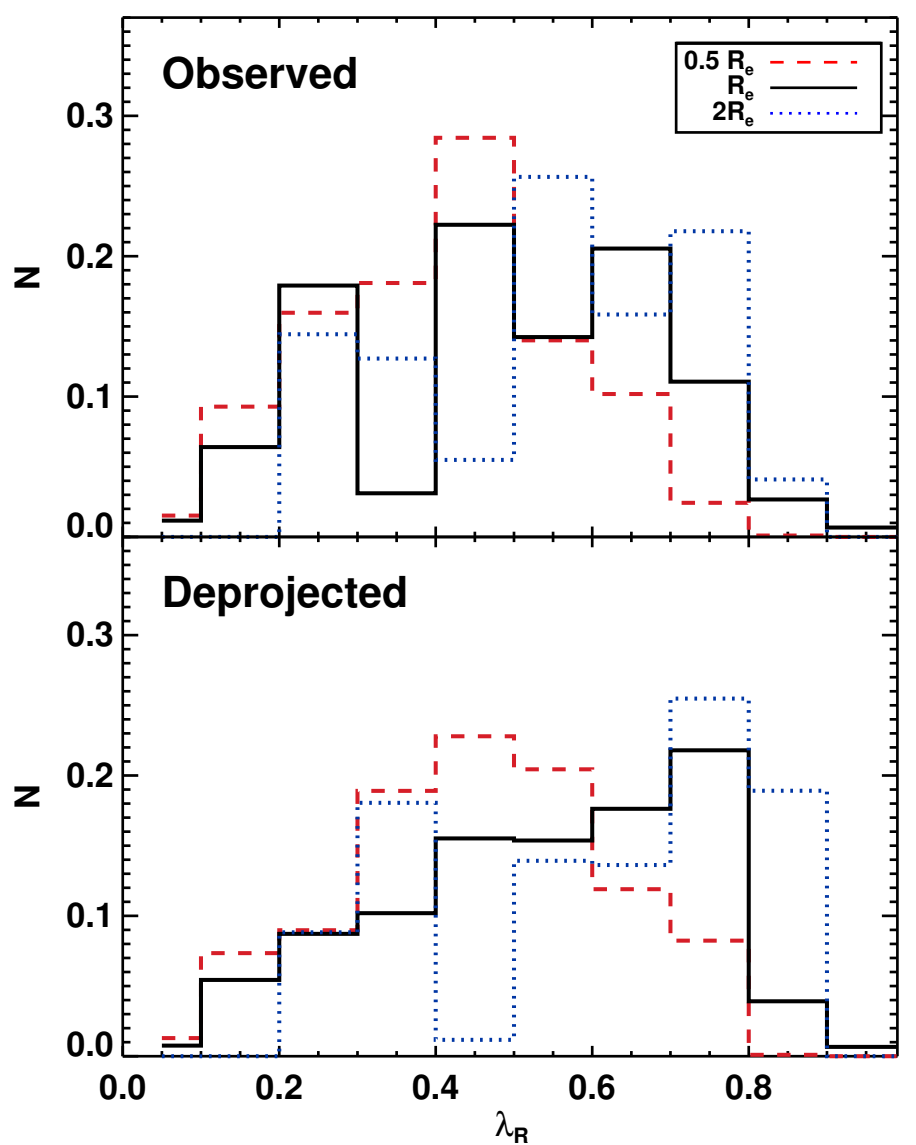

Fig. 1. Volume-corrected $\lambda_{\mathrm{R}}$ distributions for different aperture sizes (i.e. $0.5 R_{\mathrm{e}}, R_{\mathrm{e}}$, and $2 R_{\mathrm{e}}$ ). Top panel: distribution of $\lambda_{\mathrm{R}}$ as observed, while bottom panel: distribution of deprojected values (as explained in Appendix A).

and tidal interactions, are minimized. This is also supported by the close correspondence between the kinematic position angle based on the stellar velocity fields and the latter PA based on the outer radii (see Fig. 2 of Barrera-Ballesteros et al. 2014). We estimated that ellipticities measured within $R_{\mathrm{e}}$ are on average $6 \%$ smaller than those used here. Nevertheless, we confirmed that there is a good correspondence between the different approaches, aside from extreme cases.

\subsection{Global values and aperture transformations}

Our dataset allows for the exploration of the specific angular momentum on different aperture sizes. The vast majority of galaxies reach $R_{\mathrm{e}}(97 \%)$, while $61 \%$ reach up to $2 R_{\mathrm{e}}$ (see Fig. 4 in FLV17 for details).

Figure 1 shows the normalised observed and deprojected $\lambda_{\mathrm{R}}$ distributions measured within $0.5 R_{\mathrm{e}}, R_{\mathrm{e}}$, and $2 R_{\mathrm{e}}$. For the eight galaxies not reaching one $R_{\mathrm{e}}$ with our $\mathrm{S} / \mathrm{N}$ requirements, we extrapolated their values up to $R_{\mathrm{e}}$ based on their integrated profiles, as this extrapolation would be relatively safe (see Fig. 3). We note that we did not attempt to extrapolate values up to $2 R_{\mathrm{e}}$, as it would be more uncertain. Deprojected $\lambda_{\mathrm{R}}$ values were obtained following the prescriptions outlined in Appendix A. In order to provide the most representative distributions for the general population of galaxies, the histograms have been computed by weighting each galaxy contribution by the volume correction factor $\left(V_{\max }^{-1}\right)$. The figure shows a mild increase in $\lambda_{\mathrm{R}}$ with the aperture size, as expected if the majority of galaxies display clear

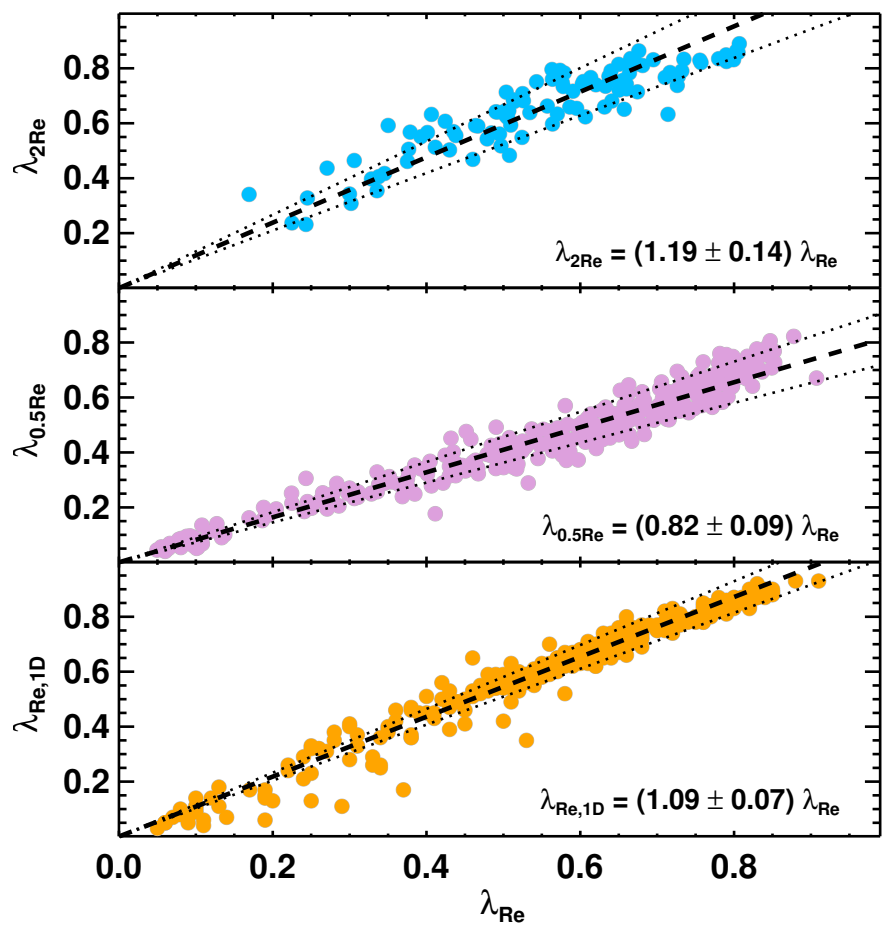

Fig. 2. Stellar angular momentum $\left(\lambda_{R}\right)$ aperture relations for different aperture sizes. Top and middle panels: relation between $\lambda_{\mathrm{Re}}$ with the values of smaller/larger apertures $0.5 R_{\mathrm{e}}$ and $2 R_{\mathrm{e}}$. Bottom panel: comparison of $\lambda_{\operatorname{Re}}$ with that computed with a 1D long-slit along the major axis of the galaxies. The dashed lines indicate the biweight mean values while dotted lines indicate the standard deviation.

rotation. While this difference may not be so obvious in the distributions of observed values, it is clearly shown in the deprojected ones, peaking at $\sim 0.45, \sim 0.75$, and $\sim 0.80$, respectively, for each aperture.

Since data reaching one $R_{\mathrm{e}}$ is not always available in other data sets, here we provide transformations between apertures based on our data. This enables us to investigate how well the measured apparent stellar angular momentum at smaller radii can be extrapolated to larger radii. In Fig. 2 we compare $\lambda_{\text {Re }}$ with $\lambda_{0.5 \mathrm{Re}}$ and $\lambda_{2 \mathrm{Re}}$ measured within half and twice the half-light radius, respectively, for those galaxies for which the kinematics extends far enough. The dashed curves represent the biweight mean relations:

$\lambda_{0.5 \mathrm{Re}}=(0.82 \pm 0.09) \lambda_{\mathrm{Re}}, \quad \lambda_{2 \mathrm{Re}}=(1.19 \pm 0.14) \lambda_{\mathrm{Re}}$,

which provide approximate extrapolations for galaxies of all Hubble types. Note that the systematic trend discussed in Fig. 1 is even more evident here. Also, the lack of low $\lambda_{\operatorname{Re}}$ and $\lambda_{2 \mathrm{Re}}$ values in the top panel highlights one of the limitations of the CALIFA target selection: large and massive nearby early-type galaxies, which are the main constituents of the slow rotator family (e.g. Emsellem et al. 2011; Veale et al. 2017b) appear in low numbers. Nevertheless, the correlations presented here are in good agreement with those presented in van de Sande et al. (2017) (e.g. $\lambda_{0.5 \mathrm{Re}} \approx 0.79 \lambda_{\mathrm{Re}}$ ).

The availability of stellar kinematic maps is rapidly increasing with ongoing and upcoming integral-field spectroscopic instruments and surveys. Even so, much of the stellar kinematic data at higher redshift will remain based on long-slit spectroscopy, which instead provides stellar velocity and dispersion profiles. Assuming the usual major-axis orientation of the long-slit, we use the kinemetry routine (Krajnović et al. 2006) to 


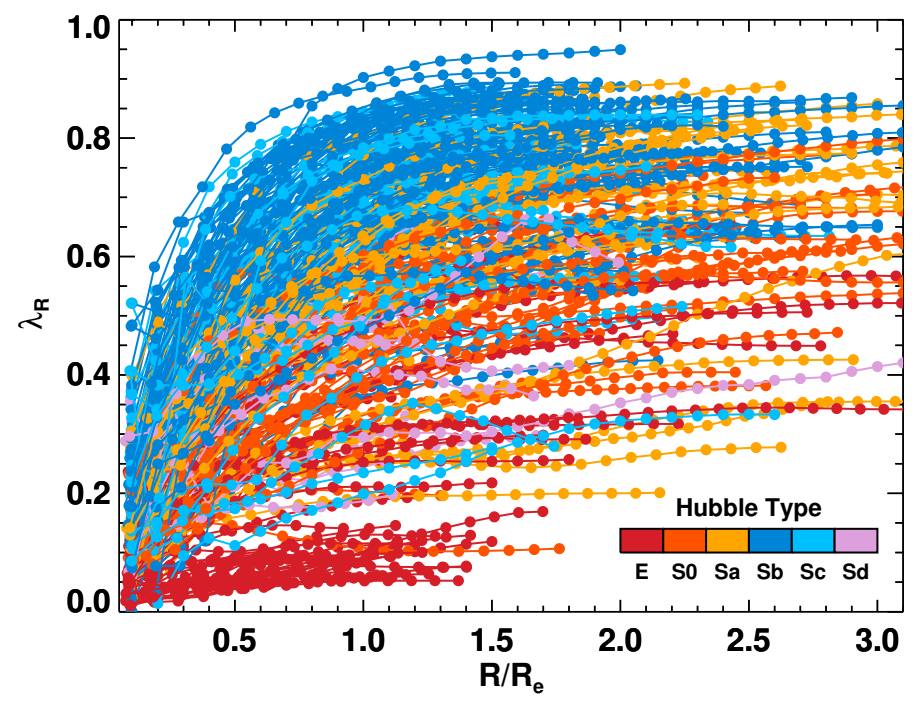

Fig. 3. Integrated $\lambda_{\mathrm{R}}$ profiles for our CALIFA sample of 300 galaxies. The profiles are normalized with $R_{\mathrm{e}}$ and color-coded by Hubble type (as indicated by the colorbar).

extract from the stellar kinematic maps of all CALIFA galaxies a major-axis rotation and dispersion profile. In the same way as Eq. (1) for $\lambda_{\mathrm{Re}}$, we then compute $\lambda_{\mathrm{Re}, 1 \mathrm{D}}$ from all radial bins out to the half-light radius $R_{\mathrm{e}}$, resulting in the correlation shown in the bottom panel of Fig. 2. The solid curve represents the biweight mean relation

$\lambda_{\mathrm{Re}, 1 \mathrm{D}}=(1.09 \pm 0.07) \lambda_{\mathrm{Re}}$.

Our relation differs somewhat from Toloba et al. (2015) (i.e. $\lambda_{\mathrm{Re}, 1 \mathrm{D}} \approx 1.56 \lambda_{\mathrm{Re}}$ ), which is likely due to differences in the size and type of galaxy samples used: 300 galaxies of all Hubble types versus 39 dwarf elliptical galaxies in the Virgo cluster. In addition, to aid in the comparison with high redshift measurements, we computed the relation between $\lambda_{\operatorname{Re}}$ and $(V / \sigma)_{\operatorname{Re}, 1 \mathrm{D}}$. As shown in Eq. (B1) of Emsellem et al. (2011), the relation between $\lambda_{\operatorname{Re}}$ and $(V / \sigma)$ has a quadratic form depending on a single parameter $\kappa$. We have fit the relation and obtained a value of $\kappa=1.1$ for all Hubble types, which is the same value derived in the ATLAS $^{3 \mathrm{D}}$ survey for early-type galaxies.

\subsection{Integrated radial profiles}

Figure 3 shows the apparent stellar angular momentum $\lambda_{\mathrm{R}}$ defined in Eq. (1) as a function of increasing enclosed radius $(R)$ along the major axis, normalized by the effective radius $R_{\mathrm{e}}$ of each galaxy. The color represents the Hubble type of the galaxy, from spheroid-dominated ellipticals in red to disk-dominated spirals in blue.

The elliptical galaxies typically have the lowest $\lambda_{R}$ values at a given (normalized) radius, even though in most cases the angular momentum does gently rise at larger radii. This is in line with the significant net rotation observed from radial velocity measurements of planetary nebulae and globular clusters in the outskirts of elliptical galaxies (e.g. Bellstedt et al. 2017). Moreover, even giant ellipticals like M 87 in the Virgo Cluster that appear round, in deeper images do show in the outskirts significant flattening reflecting at least partial rotational support (e.g. Liu et al. 2005). Additional evidence is found in early-type galaxies with faint spiral-like structures found at large radii (e.g. Gomes et al. 2016). Our findings are consistent with dedicated studies of early-type galaxies reaching up to $5 R_{\mathrm{e}}$ (e.g. Raskutti et al. 2014; Boardman et al. 2017).

Rather unexpected is that the galaxies which have the $\lambda_{\mathrm{R}}$ profiles with the largest amplitudes are not the most disk-dominated spiral galaxies. Already in the inner parts, the stars in Sb galaxies have a larger apparent angular momentum than S0 and Sa galaxies, as anticipated from the larger disk-to-total fractions of $\mathrm{Sb}$ compared to $\mathrm{S} 0 / \mathrm{Sa}$ galaxies. However, the $\lambda_{\mathrm{R}}$ values of $\mathrm{Sb}$ galaxies are on average also significantly higher than for Sc and $\mathrm{Sd}$ galaxies even though the latter are relatively more disk dominated. The most extreme cases in our sample are MCG-02-51004 (ID: 868), NGC 6301 (ID: 849), and UGC 12518 (ID: 910). See Table B. 1 for details.

Since $\lambda_{\mathrm{R}}$, as opposed to $V / \sigma$, is normalized in Eq. (1) by the sum of the squares of velocity $(V)$ and dispersion $(\sigma)$, it not only has a well-defined maximum of unity, but should also be nearly independent of mass - the enclosed total mass is namely proportional to the second velocity moment, which after projection and integration along the line of sight, in turn is proportional to $V^{2}+\sigma^{2}$. Therefore, the difference in $\lambda_{\mathrm{R}}$ profiles between galaxies of different morphological type can not merely be the result of a possible difference in mass.

\section{Rotation versus pressure support: $(V / \sigma, \varepsilon)$ and $\left(\lambda_{\mathrm{Re}}, \varepsilon\right)$ diagrams}

From earlier studies of E/S0 galaxies, in particular from the SAURON project (Emsellem et al. 2007; Cappellari et al. 2007) and ATLAS ${ }^{3 D}$ Survey (Emsellem et al. 2011), we know that the slow-rotator and fast-rotator galaxies, apart from their different position in the $\left(\lambda_{\mathrm{Re}}, \varepsilon\right)$-diagram, do have other distinct properties. Slow rotators often show kpc-scale kinematically distinct cores (KDCs) with similarly-old ages as the rest of the stars in a galaxy that tends to be a quiescent, massive elliptical galaxy with a mildly triaxial intrinsic shape (e.g McDermid et al. 2006). Most elliptical galaxies and nearly all lenticular galaxies are, however, fast rotators having an intrinsic shape, apart from the common presence of bars, that is fully consistent with oblate axisymmetry and spanning a wide range in flattening. They show regular rotation with aligned photometric and kinematic axes even though a KDC is sometimes present, but typically of smaller scale than in slow rotators and containing stars that are on average younger than the main body. A similar picture is revealed by the E (red) and S0 (orange) galaxies from the CALIFA survey.

Figure 4 shows both the more traditional ordered-overrandom stellar motion $(V / \sigma)_{\mathrm{e}}$ (left panel) and apparent stellar angular momentum $\lambda_{\mathrm{Re}}$ (right panel) as function of the ellipticity $\varepsilon$. The solid curve indicates the demarcation line between slowrotator and fast-rotator galaxies as inferred from the ATLAS ${ }^{3 \mathrm{D}}$ survey of elliptical $(E)$ and lenticular (S0) galaxies. The CALIFA survey explores in a homogeneous way galaxies of all Hubble types, as indicated by the color of the symbols. The Sa and $\mathrm{Sb}$ galaxies show the expected continuation of fast-rotator $\mathrm{E} / \mathrm{S} 0$ galaxies: reaching higher values of $(V / \sigma)_{\mathrm{e}}$ and $\lambda_{\mathrm{Re}}$ and having on average larger $\varepsilon$, consistent with the increasing dominance of a disk with resulting increase in rotational support and flattening. Interestingly though, the rotational support is decreasing again with $\mathrm{Sc}$ and in particular Sd galaxies, some of which reach $\lambda_{\mathrm{Re}}$ values close or even below the slow-fast-rotator demarcation line. Still, they remain very different from slow-rotator elliptical galaxies because the spiral galaxies have much larger $\varepsilon$ values and hence are intrinsically much flatter. We explore this behavior in more detail in the next section (Sect. 4.1). 

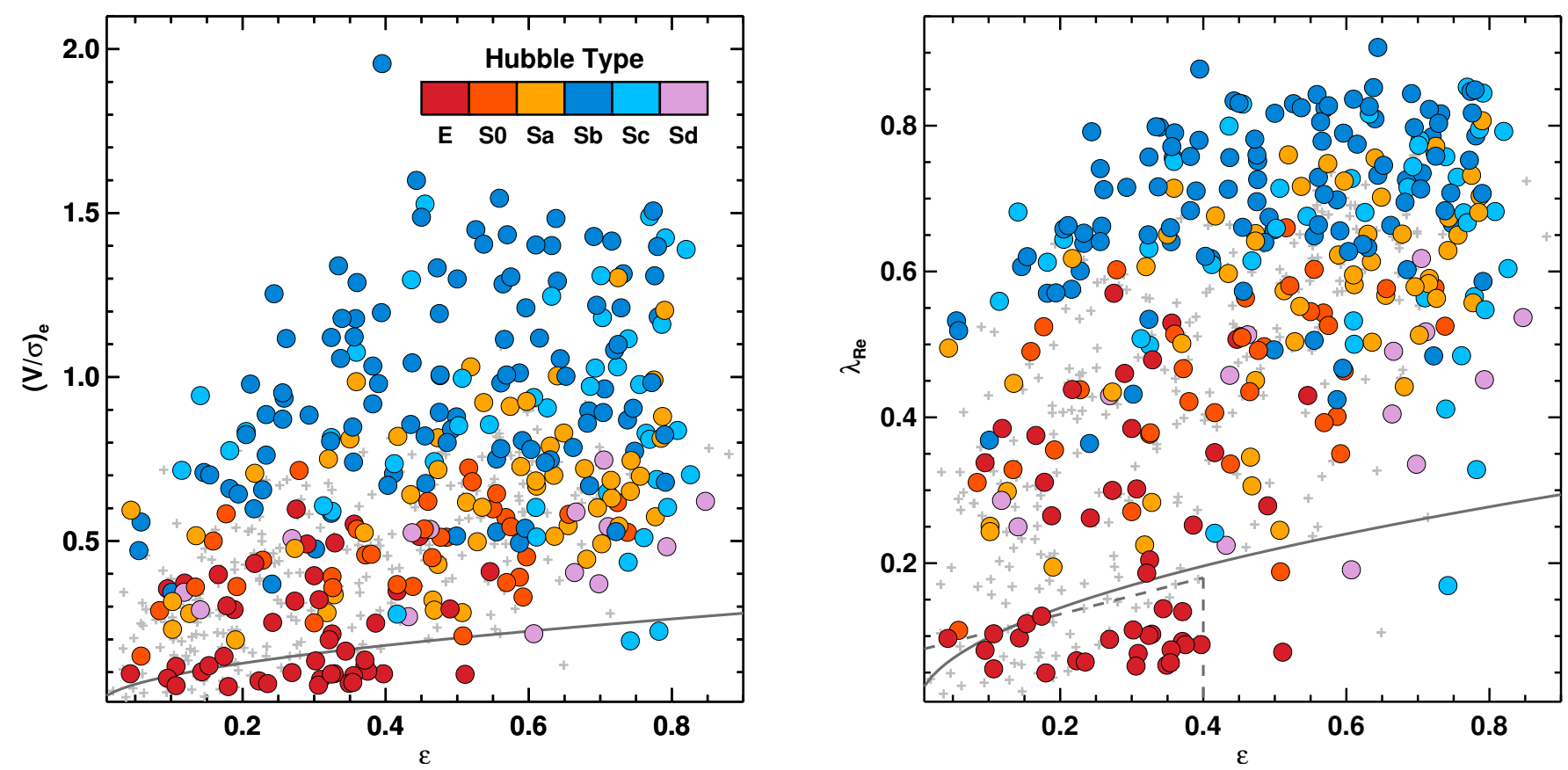

Fig. 4. $(V / \sigma, \varepsilon)_{\mathrm{e}}(l e f t)$ and $\left(\lambda_{\mathrm{Re}}, \varepsilon\right)(r i g h t)$ relations for the CALIFA sample of 300 galaxies. Galaxies are color-coded with Hubble type as indicated by the colorbar. For reference, we plot the ATLAS ${ }^{3 \mathrm{D}}$ sample with gray crosses. The solid line demarcates the division between Slow and Fast rotators as established by Emsellem et al. (2011). The dashed line in the right panel marks the revised division between Slow and Fast rotators defined by Cappellari (2016).

We chose to plot in Fig. 4 the ATLAS ${ }^{3 \mathrm{D}}$ sample for reference, as it provides values for both $(V / \sigma)_{\mathrm{e}}$ and $\lambda_{\mathrm{Re}}$. The comparison of CALIFA with ATLAS ${ }^{3 \mathrm{D}}$ and other samples in the literature is good overall. While differences in the range of measured ellipticities are small, the biggest discrepancies appear in the range of $\lambda_{\operatorname{Re}}$ values. Differences with C16 and vdS17 are mostly on the maximum values of $\lambda_{\mathrm{Re}}$ reached. While our largest values are around $\lambda_{\mathrm{Re}} \sim 0.85$, the SAMI survey galaxies hardly go over 0.8 . This is in contrast with the MaNGA sample of G18 that displays $\lambda_{\operatorname{Re}}$ values that often reach (and extend past) the theoretical maximum of 1.0. As opposed to G18 galaxies, our sample lacks round, fast rotating galaxies, which may be due to the CALIFA sample selection that precludes the inclusion of large, faceon disks. Interestingly, the range of $\lambda_{\operatorname{Re}}$ values of Sánchez et al. (2018) and Fischer et al. (2019), also based on MaNGA data, is consistent with the ones presented here. The sometimes extreme particularities of the beam corrections applied in G18 as opposed to Sánchez et al. (2018) and Fischer et al. (2019) may be at the heart of the large differences between the two studies on the same dataset. The similar effect is also true for when comparing our sample with that of vdS17. In this particular case, differences can be due to the particular definition the SAMI team adopted for $R_{j}$ in Eq. (1) (e.g. semi-major axis of the ellipse on which spaxel $j$ lies, instead of the circular projected radius to the center). This also results in a lower $\lambda_{\text {Re }}$ value as compared to the Polar $\mathrm{R}_{j}$ definition that is adopted here. Regardless of the specific details in the sample selection and peculiarities in the $\lambda_{\operatorname{Re}}$ calculation of the three surveys, they are largely complementary.

\subsection{Trends with global parameters}

To investigate further the properties of galaxies of all morphological types in the $\left(\lambda_{\mathrm{Re}}, \varepsilon\right)$-diagram, we show in Fig. 5 the relation between $\lambda_{\mathrm{Re}}$ and different global parameters, color-coding galaxies according to their Hubble type.
The top row in Fig. 5 shows the behavior of $\lambda_{\text {Re }}$ with Hubble type, $r$-band absolute magnitude and total stellar mass (from left to right). Not surprisingly the ellipticals display a wide range of $\lambda_{\operatorname{Re}}$ values, from the lowest in the sample close to zero to almost 0.6. As originally observed in the SAURON survey, the $\mathrm{E}$ family comprises galaxies that includes both slow and fast rotators. The middle and right panels confirm that luminosity and mass are the best predictors for slow rotators, being the dominant population at the high luminosity and mass end. Nevertheless, the increase of $\lambda_{\operatorname{Re}}$ with Hubble type would still hold even if slow rotators were not considered. This increasing trend with morphological type was already observed by C16 in the SAMI survey. Interestingly, though, our sample shows a maximum in $\lambda_{\mathrm{Re}}$ for the $\mathrm{Sb}$ galaxies, with decreasing values for later-types. We have used the much larger MaNGA sample of G18 to check this trend. While there is indeed a turning point at similar stellar masses, this is much milder than observed in our CALIFA sample at low masses. We attribute the difference to the peculiarities of our sample, which is not complete for the low luminosity (and thus mass) end (see FLV17 for more details).

The bottom row shows the relation between $\lambda_{\mathrm{Re}}$ with $u-r$ Petrosian color, concentration index $\left(R_{90} / R_{50}\right.$, measured as the ratio of 90 and 50 percentile Petrosian radius), and star-formation rates derived from $\mathrm{H} \alpha$ emission line fluxes in Sánchez et al. (2017). Our sample of E/S0/Sa galaxies show a well-defined vertical sequence around the same red color, while later types display bluer colors. The middle panel shows that, slow-rotators aside, the concentration index is clearly related to $\lambda_{\mathrm{Re}}$ with larger values reached by the $\mathrm{Sb}$ types. This is expected as light concentration is a proxy for the bulge(+bar)-to-total ratio, which in turn is the main driver behind the Hubble morphological classification (e.g. Shimasaku et al. 2001; Strateva et al. 2001). Still, there appears to be some scatter in the relation, consistent with that shown already in C16. The family of $\mathrm{Sb}$ galaxies also appear to be the currently higher 

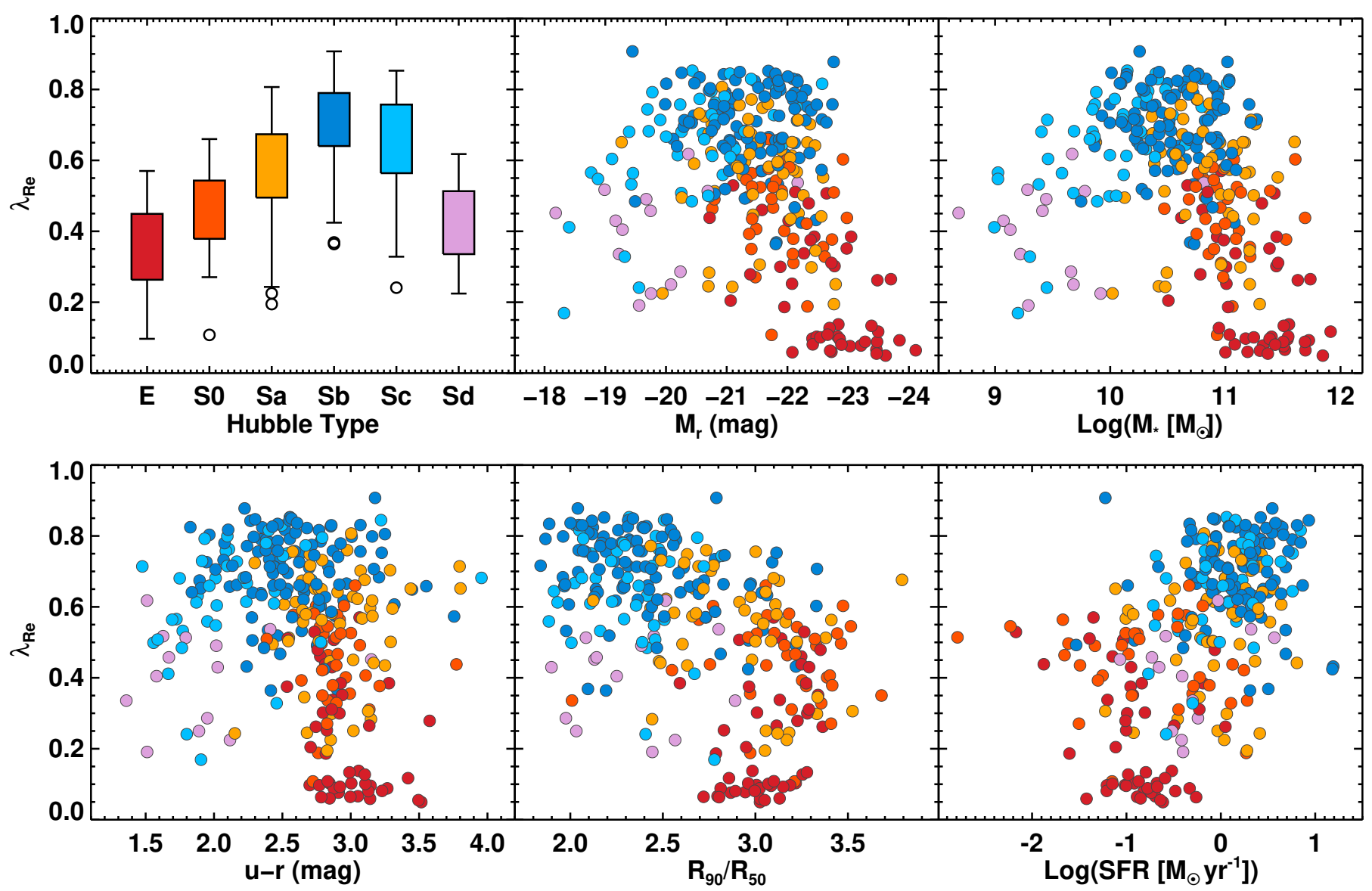

Fig. 5. $\lambda_{\mathrm{Re}}$ relations with global parameters for the sample of 300 CALIFA galaxies. Galaxies are color-coded with Hubble type. Top left panel: whisker plot enclosing the interquartile range (IQR), defined at IQR25\%-IQR75\% for galaxies of each morphological type. The whiskers extend out to the maximum or minimum value of the data, or to 1.5 times IQR25\% or IQR75\% in case there is data beyond this range. Outliers are identified with small circles. Other panels: relations with $r$-band absolute magnitude $M_{r}$, total stellar mass $M_{\star}, u-r$ Petrosian color, concentration index $R_{90} / R_{50}$, and star formation rate SFR. See text for details.

star-forming systems (right panel). Similarly, going from spiral to elliptical galaxies, the SFR decreases, so it also unsurprising to find the trend of decreasing $\lambda_{\mathrm{R}}$ with lower SFR.

The $\lambda_{\mathrm{Re}}$ values found for the galaxies in our sample confirm the predominance of Slow rotators in high-mass, highluminosity systems. We estimate an overall fraction of $28 \%$ of Slow rotators with stellar masses above $10^{11} M_{\odot}$, based on the Emsellem et al. 2011 definition. This number sits in the middle of the wide range of predictions provided by the latest surveys (e.g. Emsellem et al. 2011; D'Eugenio et al. 2013; Fogarty et al. 2014; Veale et al. 2017a; van de Sande et al. 2017) which display values between $15 \%$ and $80 \%$ for masses above $10^{11} M_{\odot}$. Our lower value is likely due to the fact that the CALIFA survey is complete only up to $10^{11.44} M_{\odot}$ (see Walcher et al. 2014, for details).

Despite the limited number of galaxies compared to other surveys, our sample shows two areas with interesting results: (i) the low $\lambda_{\mathrm{Re}}$ values for the late-type spirals, and (ii) the particular properties of the fastest rotators.

\subsubsection{Low $\lambda_{\operatorname{Re}}$ spirals}

We have investigated the reasons for the surprisingly low $\lambda_{\operatorname{Re}}$ values observed in the latest-type galaxies and found two potential explanations. There is a group of $\mathrm{Sc} / \mathrm{Sd}$ galaxies with $\lambda_{\mathrm{Re}}$ values below 0.35 . We have checked and these are both irregular or fairly face-on systems. This naturally explains their unusual location in the $\left(\lambda_{\mathrm{Re}}, \varepsilon\right)$ diagram. The same feature was found by Graham et al. (2018) in their much larger sample of galaxies. The remaining group of $\mathrm{Sc} / \mathrm{Sd}$ galaxies with $\lambda_{\mathrm{Re}}$ values between 0.35 and 0.6 are typically edge-on systems. We have explored whether they present large extinction values, as dust obscuration could prevent the full integration of the stellar kinematics along the line-of-sight and thus led to lower rotation amplitudes. Displaying the bluest colors of the entire sample, this option does not seem to be likely. This is confirmed by the much more detailed study of the extinction in the CALIFA galaxies by González Delgado et al. (2015). We note, however, that simulations suggest that observational estimates could be underestimated for this kind of systems (see Ibarra-Medel et al. 2019, for details). In addition, we have also checked that those galaxies display velocity dispersions well above the point where the limits in the CALIFA spectral resolution are an issue (see Fig. 9 in FLV17).

The large observed $\varepsilon$ values for those $\mathrm{Sc} / \mathrm{Sd}$ galaxies imply that we need a way to keep their dynamically hot stellar disk geometrically thin. We note that they contain small bulges (as observed by their low concentration values), and also are not the highest star-forming galaxies. We postulate that the presence of a relatively large dark matter halo provides an additional vertical force to keep the disk geometrically thin while being dynamically heated. Our initial assessment, based on 
dynamical models of our sample (Zhu et al. 2018), suggests an enclosed mass that is up to a factor ten larger than the estimated baryonic (stellar plus gas) mass already within the halflight radius. A preliminary confirmation of this was presented in Fig. 3 of Falcón-Barroso et al. (2015). This is in line with previous results in the literature presenting evidence of "thicker" thin disks in late-type spirals (e.g. Yoachim \& Dalcanton 2006; Comerón et al. 2011).

\subsubsection{Fastest rotators}

We have identified a group of 19 galaxies with $\lambda_{\mathrm{Re}} \geq 0.82$ (i.e. the top $5 \%$ of the distribution). They are mostly $\mathrm{Sb} / \mathrm{Sc}$ galaxies. In Fig. 5 they happen to have intermediate absolute magnitudes, masses, and colors. They are not special in any of those three parameters with respect to other galaxies with lower $\lambda_{\mathrm{Re}}$ values. They are peculiar in that they are the highest star-forming galaxies with some of the smallest bulges, as probed by the concentration index ${ }^{1}$. We have identified at least three other properties that make these galaxies unique. As shown in Fig. 6, they tend to have the largest sizes at a given stellar mass, display some of the strongest average luminosity-weighted inner age gradients measured by García-Benito et al. (2017), and also appear to have rather homogeneous mean stellar population ages within $R_{\mathrm{e}}$ of about 1 Gyr (González Delgado et al. 2015). We inspected for any dependence with environment, either local or global, and found no significant trends.

The relative difference between the observed $\lambda_{\operatorname{Re}}$ values of this group of galaxies (see top, left panel of Fig. 5) with respect to the S0 population, raises the question whether they could fade into lenticular galaxies. Decades after the discovery of the morphology-density relation (Dressler et al. 1987), the interest in this topic has been revived by the recent results from different groups (e.g. Bedregal et al. 2006; Laurikainen et al. 2010; Kormendy \& Bender 2012; Brough et al. 2017; Greene et al. 2017) confirming the initial result, but recasting the observed phenomenon from the Slow/Fast rotator perspective (e.g. Cappellari et al. 2011b). We refer the reader to the extended review on the topic presented in Cappellari (2016). At first sight, based purely on $\lambda_{\mathrm{Re}}$, our results suggest that the transformation between Sa galaxies into S0s is possible. We note, however, that in a fading scenario, both stellar mass and $\lambda_{\mathrm{Re}}$ are expected to be conserved. This seems more difficult in the case of Sb and Sc galaxies, for which the difference with respect to the lenticulars in $\lambda_{\mathrm{Re}}$ is significant. For Sd galaxies, even though $\lambda_{\mathrm{Re}}$ values are consistent with those of lenticulars, their light concentrations are much lower and thus it seems unlikely they will fade into lenticulars with typically much larger bulge-to-disk ratios. Furthermore, the amount of mass in gas for these late-type galaxies can be up to $50 \%$ of their baryonic total mass (e.g. Papastergis et al. 2012), making it very difficult to turn all that matter into stars by fading within a Hubble time without substantially increasing the total mass budget of the system. Pre-processing in groups, with tidal interactions and/or major mergers seem to be more likely mechanisms (e.g. Querejeta et al. 2015).

\subsection{Trends in the $S F R-M_{\star}$ diagram}

An interesting way of looking at variations of $\lambda_{\operatorname{Re}}$ is through the extensively studied star formation rate-stellar mass relation (e.g.

\footnotetext{
1 This is confirmed by the detailed bulge/disk photometric decomposition of Méndez-Abreu et al. (2017) for the limited subset of CALIFA galaxies in common with the sample presented here.
}
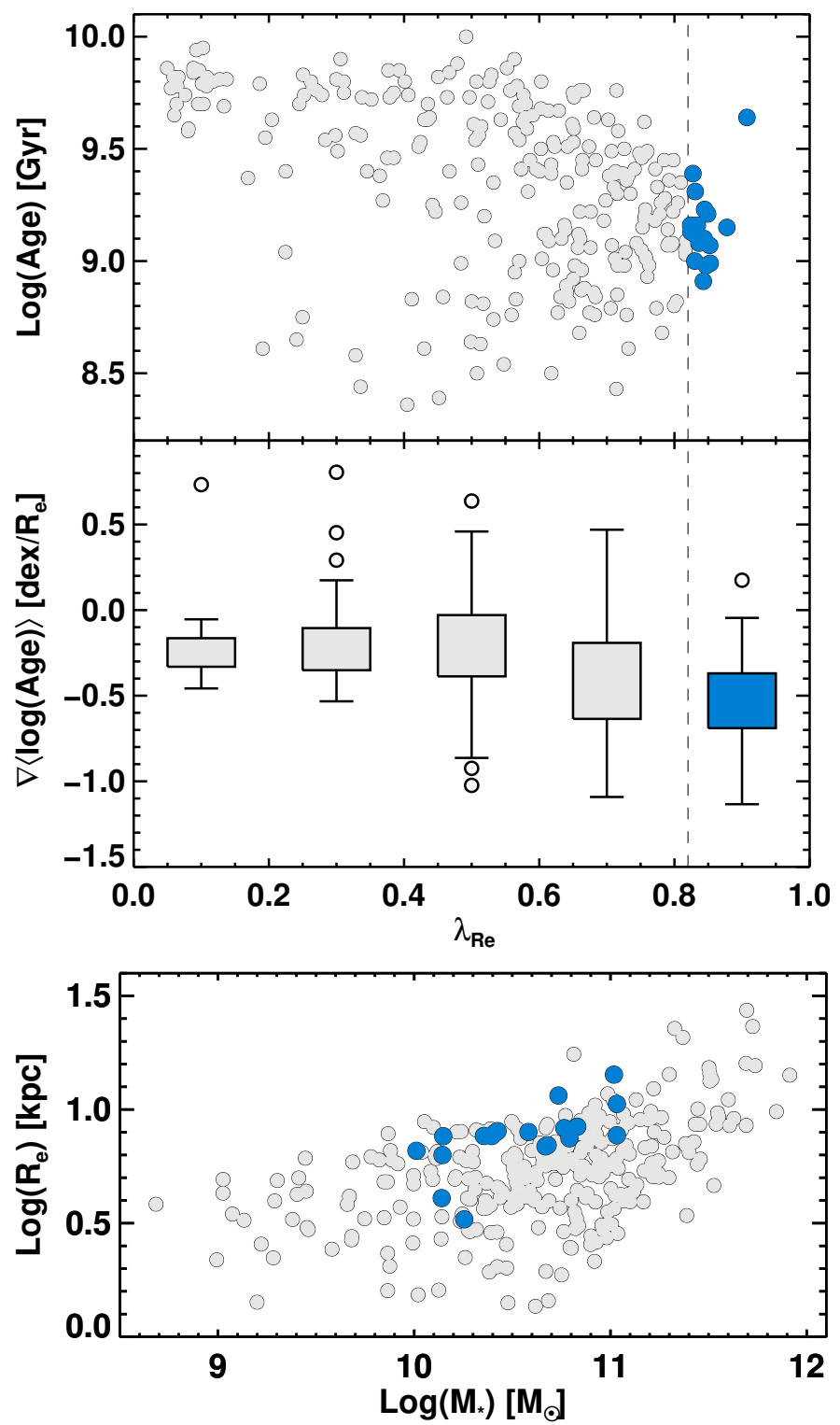

Fig. 6. Top and middle panels: $\lambda_{\mathrm{Re}}$ relations with mean age within $R_{\mathrm{e}}$, mean stellar age gradient within $R_{\mathrm{e}}$ for our sample of CALIFA galaxies. Bottom panel: stellar mass-size relation. The whisker plot in the middle panel was computed in the same way as in Fig. 5. Galaxies above the 95\% percentile of $\lambda_{\mathrm{Re}}$ distribution of the sample are marked in blue. The dashed line in the top and middle panels marks that percentile.

Croton et al. 2006; Cortese et al. 2019), shown in Fig. 7 for our sample. We have divided the diagram in bins of SFR and stellar mass. Each subpanel presents the $\left(\lambda_{\mathrm{Re}}, \varepsilon\right)$ relation for that bin, showing in gray all CALIFA galaxies and highlighting in color those belonging to that bin with their Hubble type. The number on the top-left corner of each inset gives the $V_{\max }^{-1}$-weighted $\lambda_{\operatorname{Re}}$ average for the highlighted galaxies in that bin.

The figure shows that the main star-forming sequence is made of galaxies with increasing $\lambda_{\mathrm{Re}}$ as both the SFR and stellar mass grow. The high-mass and high star-forming region is populated by $\mathrm{Sa} / \mathrm{Sb}$ galaxies mostly, whereas the low SFR and stellar mass ends are dominated by later-type galaxies $(\mathrm{Sc} / \mathrm{Sd})$. As already highlighted by numerous studies, galaxies departing from the main star-forming sequence are mostly early-type Es and S0s (e.g. Schawinski et al. 2014). It appears that the dynamically coldest disks are found in the most massive and 


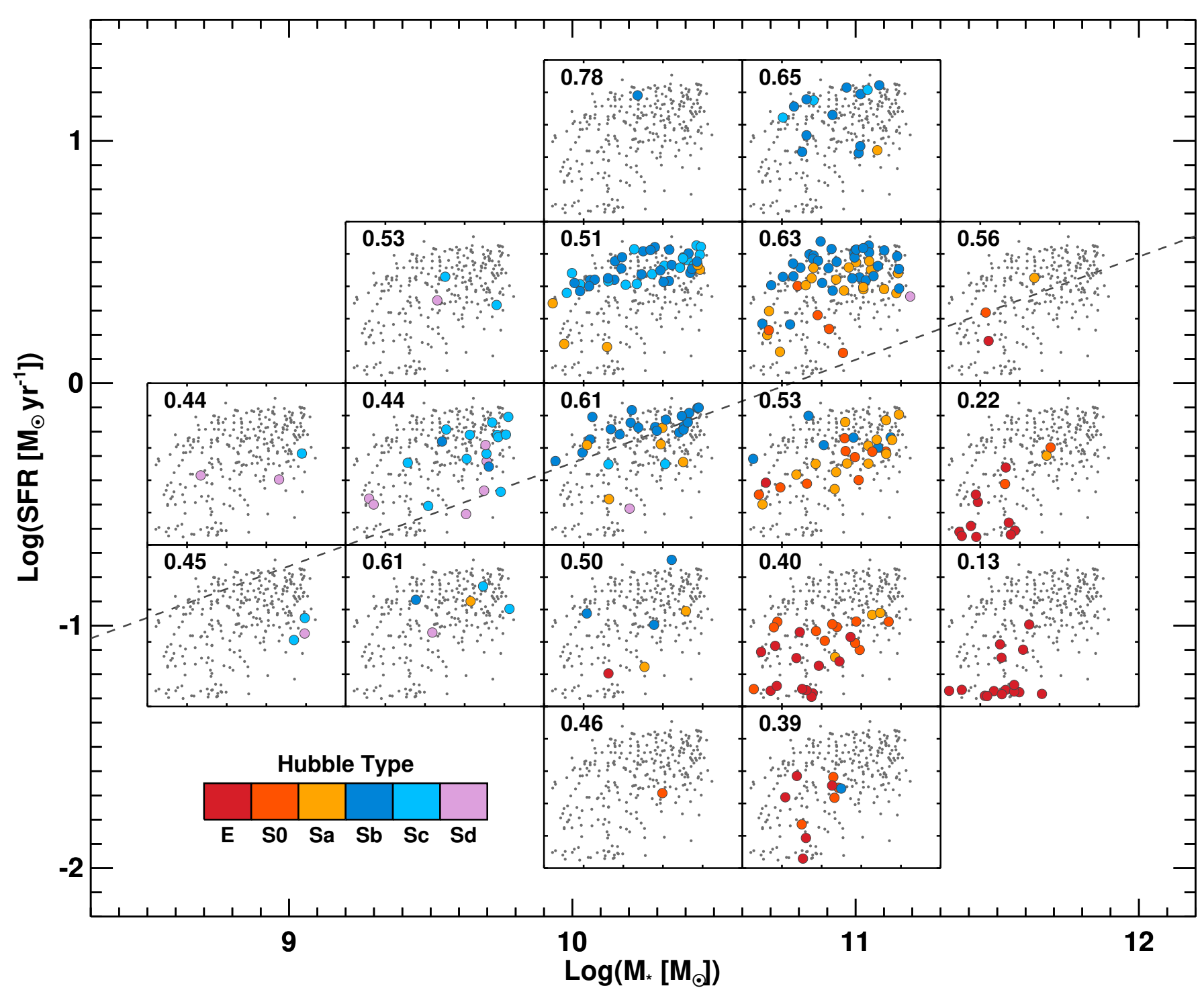

Fig. 7. $\lambda_{\mathrm{Re}}-\epsilon$ relation as a function of location in the star-formation rate versus total stellar mass $\left(M_{\star}\right)$ relation for the CALIFA galaxies. Each panel shows the complete sample highlighting in color the ones belonging to each specific SFR $-M_{\star}$ bin. The number in each subpanel gives the $V_{\max }^{-1}$-weighted $\lambda_{\mathrm{Re}}$ average for the highlighted galaxies in that bin. The dark gray dashed line in the SFR versus $M_{\star}$ on the right marks the division between the main star-forming sequence and quiescent galaxies from Renzini \& Peng (2015).

more actively star-forming systems (e.g. Catalán-Torrecilla et al. 2017; Méndez-Abreu et al. 2019). The trends with $\lambda_{\text {Re }}$ observed here are supported by a similar analysis with the EAGLE cosmological simulation (Walo-Martín et al., in prep.).

\section{Conclusions}

This paper presents the CALIFA view on the stellar angular momentum distribution for a sample of 300 galaxies across the Hubble sequence. Our dataset allows us to study the distribution of $\lambda_{\mathrm{R}}$ for different apertures $\left(0.5 R_{\mathrm{e}}, R_{\mathrm{e}}, 2 R_{\mathrm{e}}\right)$ and provides the relationship between them, including conversions to $\lambda_{\mathrm{R}}$ computed with a long-slit along the major axis of the galaxies. Our sample also helps us to investigate the relationship between $\lambda_{\mathrm{R}}$ and different global properties of galaxies (e.g. Hubble type, absolute magnitude, $u-r$ color, concentration index, stellar mass, and star formation rate).

In addition, we analyze the distribution of galaxies in the classical $(V / \sigma, \varepsilon)$ and $\left(\lambda_{\mathrm{Re}}, \varepsilon\right)$ diagrams, often used to study the level of rotation over pressure support in galaxies. Our results for early-type (E and S0) galaxies are consistent with previous studies in the literature for the same kind of galaxies. The extension to later-types ( $\mathrm{Sa}$ to $\mathrm{Sd}$ ) provided by our sample presents two interesting results. On one side, we find a maximum $\lambda_{\mathrm{Re}}$ of around $\sim 0.85$ for large, relatively massive and highly starforming galaxies (typically $\mathrm{Sb}$ systems). On the other hand, rather unexpectedly, we observe relatively low $\lambda_{\operatorname{Re}}$ values for low-mass $\mathrm{Sc} / \mathrm{Sd}$ systems. We will explore these two areas in forthcoming papers for a broader discussion of the nature of S0 galaxies and to investigate the dark matter content of low mass systems.

The results presented here with the CALIFA sample in terms of the stellar angular momentum distribution of galaxies are just the tip of the iceberg of possibilities for extending our understanding of galaxy formation and evolution. Ongoing large surveys have already started to make use of this information in different areas (e.g. vdS17), with a boost in this field coming with the measurement of $\lambda_{\mathrm{Re}}$ for thousands of galaxies provided by the MaNGA survey team (e.g. G18). Complementarily, the first studies to relate the radial dependence of $\lambda_{R}$ to the evolution of 
galaxies are appearing in the literature (e.g. Graham et al. 2017). In the absence of high-quality observations of stellar kinematics for substantial samples of high-redshift galaxies (e.g. $z>1.0$ ), cosmological numerical simulations will allow us to explore the evolution of angular momentum as a function of cosmic time (e.g. Lagos et al. 2018; Schulze et al. 2018; Pillepich et al. 2019; van de Sande et al. 2019).

Acknowledgements. We would like to thank the anonymous referee for constructive comments that helped improve some aspects of the original manuscript. This study makes use of the data provided by the Calar Alto Legacy Integral Field Area (CALIFA) survey (http://www. califa.caha.es). Based on observations collected at the Centro Astronòmico Hispano Alemán (CAHA) at Calar Alto, operated jointly by the Max-Planck-Institut für Astronomie and the Instituto de Astrofisica de Andalucia (CSIC). CALIFA is the first legacy survey being performed at Calar Alto. The CALIFA collaboration would like to thank the IAA-CSIC and MPIA-MPG as major partners of the observatory, and CAHA itself, for the unique access to telescope time and support in manpower and infrastructures. The CALIFA collaboration thanks also the CAHA staff for the dedication to this project. Funding and financial support acknowledgements: J F.-B. from grant AYA2016-77237-C3-1-P from the Spanish Ministry of Economy and Competitiveness (MINECO); GvdV acknowledges funding from the European Research Council (ERC) under the European Union's Horizon 2020 research and innovation programme undergrant agreement no. 724857 (Consolidator Grant ArcheoDyn). B. G.-L. acknowledge support from the State Research Agency (AEI) of the Spanish Ministry of Science, Innovation and Universities (MCIU) and the European Regional Development Fund (FEDER) under grant with reference AYA2015-68217-P. SFS is grateful for the support of a CONACYT grant CB-285080 and FC-2016-01-1916, and funding from the PAPIITDGAPA-IA101217 (UNAM) project. L. Z. acknowledges support from Shang hai Astronomical Observatory, Chinese Academy of Sciences under grant no. Y895201009. L. G. was supported in part by the US National Science Foundation under Grant AST-1311862. RGD from AYA2016-77846-P, AYA2014-57490-P, AYA2010-15081, and Junta de Andalucí a FQ1580. IM from grants AYA2013 42227-P and AYA2016-76682-C3-1-P. RGB, RMGD, IM, and EP acknowledge financial support from the State Agency for Research of the Spanish MCIU through the "Center of Excellence Severo Ochoa" award to the Instituto de Astrofísica de Andalucía (SEV-2017-0709).

\section{References}

Abazajian, K. N., Adelman-McCarthy, J. K., Agüeros, M. A., et al. 2009, ApJS, 182,543

Amorín, R., Fontana, A., Pérez-Montero, E., et al. 2017, Nat. Astron., 1, 0052 Amorisco, N. C., Evans, N. W., \& van de Ven, G. 2014, Nature, 507, 335 Arnold, J. A., Romanowsky, A. J., Brodie, J. P., et al. 2014, ApJ, 791, 80 Barrera-Ballesteros, J. K., Falcón-Barroso, J., García-Lorenzo, B., et al. 2014, A\&A, 568, A70

Battaglia, G., Helmi, A., Tolstoy, E., et al. 2008, ApJ, 681, L13

Bedregal, A. G., Aragón-Salamanca, A., \& Merrifield, M. R. 2006, MNRAS, 373, 1125

Bellstedt, S., Forbes, D. A., Foster, C., et al. 2017, MNRAS, 467, 4540

Binney, J. 2005, MNRAS, 363, 937

Boardman, N. F., Weijmans, A.-M., van den Bosch, R., et al. 2017, MNRAS, 471, 4005

Brasseur, C. M., Martin, N. F., Macciò, A. V., Rix, H.-W., \& Kang, X. 2011, ApJ, 743, 179

Brough, S., van de Sande, J., Owers, M. S., et al. 2017, ApJ, 844, 59

Bullock, J. S., Dekel, A., Kolatt, T. S., et al. 2001, ApJ, 555, 240

Bundy, K., Bershady, M. A., Law, D. R., et al. 2015, ApJ, 798, 7

Burkert, A., Förster Schreiber, N. M., Genzel, R., et al. 2016, ApJ, 826, 214

Cappellari, M. 2016, ARA\&A, 54, 597

Cappellari, M., \& Copin, Y. 2003, MNRAS, 342, 345

Cappellari, M., \& Emsellem, E. 2004, PASP, 116, 138

Cappellari, M., Emsellem, E., Bacon, R., et al. 2007, MNRAS, 379, 418

Cappellari, M., Emsellem, E., Krajnović, D., et al. 2011a, MNRAS, 413, 813

Cappellari, M., Emsellem, E., Krajnović, D., et al. 2011b, MNRAS, 416, 1680

Catalán-Torrecilla, C., Gil de Paz, A., Castillo-Morales, A., et al. 2017, ApJ, 848, 87

Cid Fernandes, R., Pérez, E., García Benito, R., et al. 2013, A\&A, 557, A86

Comerón, S., Elmegreen, B. G., Knapen, J. H., et al. 2011, ApJ, 741, 28

Cortese, L., Fogarty, L. M. R., Bekki, K., et al. 2016, MNRAS, 463, 170

Cortese, L., van de Sande, J., Lagos, C. P., et al. 2019, MNRAS, 485, 2656

Croom, S. M., Lawrence, J. S., Bland-Hawthorn, J., et al. 2012, MNRAS, 421, 872
Croton, D. J., Springel, V., White, S. D. M., et al. 2006, MNRAS, 365, 11 de Zeeuw, P. T., Bureau, M., Emsellem, E., et al. 2002, MNRAS, 329, 513 del Moral-Castro, I., García-Lorenzo, B., Ramos Almeida, C., et al. 2019, MNRAS, 485, 3794

D’Eugenio, F., Houghton, R. C. W., Davies, R. L., \& Dalla Bontà, E. 2013, MNRAS, 429, 1258

Dressler, A., Lynden-Bell, D., Burstein, D., et al. 1987, ApJ, 313, 42

Emsellem, E., Cappellari, M., Krajnović, D., et al. 2007, MNRAS, 379, 401

Emsellem, E., Cappellari, M., Krajnović, D., et al. 2011, MNRAS, 414, 888

Falcón-Barroso, J., Lyubenova, M., \& van de Ven, G. 2015, in Galaxy Masses as Constraints of Formation Models, eds. M. Cappellari, \& S. Courteau, IAU Symp., 311, 78

Falcón-Barroso, J., Lyubenova, M., van de Ven, G., et al. 2017, A\&A, 597, A48

Fischer, J. L., Domínguez Sánchez, H., \& Bernardi, M. 2019, MNRAS, 483, 2057

Fogarty, L. M. R., Scott, N., Owers, M. S., et al. 2014, MNRAS, 443, 485 García-Benito, R., González Delgado, R. M., Pérez, E., et al. 2017, A\&A, 608, A27

Gomes, J. M., Papaderos, P., Vílchez, J. M., et al. 2016, A\&A, 585, A92

González Delgado, R. M., Cerviño, M., Martins, L. P., Leitherer, C., \& Hauschildt, P. H. 2005, MNRAS, 357, 945

González Delgado, R. M., García-Benito, R., Pérez, E., et al. 2015, A\&A, 581, A103

Graham, A. W., Janz, J., Penny, S. J., et al. 2017, ApJ, 840, 68

Graham, M. T., Cappellari, M., Li, H., et al. 2018, MNRAS, 477, 4711

Greene, J. E., Leauthaud, A., Emsellem, E., et al. 2017, ApJ, 851, L33

Husemann, B., Jahnke, K., Sánchez, S. F., et al. 2013, A\&A, 549, A87

Ibarra-Medel, H. J., Avila-Reese, V., Sánchez, S. F., González-Samaniego, A. R., \& Rodríguez-Puebla, A. 2019, MNRAS, 483, 4525

Kelz, A., Verheijen, M. A. W., Roth, M. M., et al. 2006, PASP, 118, 129

Kormendy, J., \& Bender, R. 2012, ApJS, 198, 2

Krajnović, D., Cappellari, M., de Zeeuw, P. T., \& Copin, Y. 2006, MNRAS, 366 787

Krajnović, D., Emsellem, E., Cappellari, M., et al. 2011, MNRAS, 414, 2923

Lagos, C. D. P., Schaye, J., Bahé, Y., et al. 2018, MNRAS, 476, 4327

Lambas, D. G., Maddox, S. J., \& Loveday, J. 1992, MNRAS, 258, 404

Laurikainen, E., Salo, H., Buta, R., Knapen, J. H., \& Comerón, S. 2010 MNRAS, 405, 1089

Liu, Y., Zhou, X., Ma, J., et al. 2005, AJ, 129, 2628

Ma, C.-P., Greene, J. E., McConnell, N., et al. 2014, ApJ, 795, 158

McDermid, R. M., Emsellem, E., Shapiro, K. L., et al. 2006, MNRAS, 373 , 906

Méndez-Abreu, J., Ruiz-Lara, T., Sánchez-Menguiano, L., et al. 2017, A\&A, 598, A32

Méndez-Abreu, J., Sánchez, S. F., \& de Lorenzo-Cáceres, A. 2019, MNRAS 488, L80

Mo, H. J., Mao, S., \& White, S. D. M. 1998, MNRAS, 295, 319

Papastergis, E., Cattaneo, A., Huang, S., Giovanelli, R., \& Haynes, M. P. 2012, ApJ, 759, 138

Pillepich, A., Nelson, D., Springel, V., et al. 2019, MNRAS, 490, 3196

Querejeta, M., Eliche-Moral, M. C., Tapia, T., et al. 2015, A\&A, 579, L2

Raskutti, S., Greene, J. E., \& Murphy, J. D. 2014, ApJ, 786, 23

Renzini, A., \& Peng, Y.-J. 2015, ApJ, 801, L29

Romanowsky, A. J., \& Fall, S. M. 2012, ApJS, 203, 17

Ryś, A., Falcón-Barroso, J., \& van de Ven, G. 2013, MNRAS, 428, 2980

Ryś, A., van de Ven, G., \& Falcón-Barroso, J. 2014, MNRAS, 439, 284

Ryś, A., Koleva, M., Falcón-Barroso, J., et al. 2015, MNRAS, 452, 1888

Sánchez, S. F., Avila-Reese, V., Hernandez-Toledo, H., et al. 2018, Rev.

Mexicana Astron. Astrofis., 54, 217

Sánchez, S. F., Kennicutt, R. C., Gil de Paz, A., et al. 2012, A\&A, 538, A8

Sánchez, S. F., García-Benito, R., Zibetti, S., et al. 2016, A\&A, 594, A36

Sánchez, S. F., Barrera-Ballesteros, J. K., Sánchez-Menguiano, L., et al. 2017, MNRAS, 469, 2121

Schawinski, K., Urry, C. M., Simmons, B. D., et al. 2014, MNRAS, 440, 889

Schulze, F., Remus, R.-S., Dolag, K., et al. 2018, MNRAS, 480, 4636

Shen, S., Mo, H. J., White, S. D. M., et al. 2003, MNRAS, 343, 978

Shimasaku, K., Fukugita, M., Doi, M., et al. 2001, AJ, 122, 1238

Strateva, I., Ivezić, Ž., Knapp, G. R., et al. 2001, AJ, 122, 1861

Toloba, E., Boselli, A., Cenarro, A. J., et al. 2011, A\&A, 526, A114

Toloba, E., Guhathakurta, P., Boselli, A., et al. 2015, ApJ, 799, 172

Toomre, A., \& Toomre, J. 1972, ApJ, 178, 623

Valdes, F., Gupta, R., Rose, J. A., Singh, H. P., \& Bell, D. J. 2004, ApJS, 152, 251

van de Sande, J., Bland-Hawthorn, J., Brough, S., et al. 2017, MNRAS, 472, 1272

van de Sande, J., Lagos, C. D. P., Welker, C., et al. 2019, MNRAS, 484, 869 
Vazdekis, A., Coelho, P., Cassisi, S., et al. 2015, MNRAS, 449, 1177 Veale, M., Ma, C.-P., Greene, J. E., et al. 2017a, MNRAS, 471, 1428 Veale, M., Ma, C.-P., Thomas, J., et al. 2017b, MNRAS, 464, 356

Walcher, C. J., Wisotzki, L., Bekeraité, S., et al. 2014, A\&A, 569, A1

Weijmans, A.-M., de Zeeuw, P. T., Emsellem, E., et al. 2014, MNRAS, 444 3340

Yoachim, P., \& Dalcanton, J. J. 2006, AJ, 131, 226

Zhu, L., Ven, G. V. D., Bosch, R. V. D., et al. 2018, Nat. Astron., 2, 233

1 Instituto de Astrofísica de Canarias, Vía Láctea s/n, 38205 La Laguna, Tenerife, Spain e-mail: jfalcon@iac.es

2 Departamento de Astrofísica, Universidad de La Laguna, 38205 La Laguna, Tenerife, Spain

${ }^{3}$ Department of Astrophysics, University of Vienna, Türkenschanzstrasse 17, 1180 Vienna, Austria

${ }^{4}$ European Southern Observatory, Karl-Schwarzschild-Str. 2, 85748 Garching b. München, Germany

5 Leibniz-Institut für Astrophysik Potsdam (AIP), An der Sternwarte 16, 14482 Potsdam, Germany

${ }^{6}$ Instituto de Astronomía, Universidad Nacional Autónoma de México, Apartado Postal 70-264, México D.F. 04510, Mexico

7 Max-Planck-Institut für Astronomie, Königstuhl 17, 69117 Heidelberg, Germany

${ }^{8}$ Instituto de Astrofísica de Andalucía (IAA/CSIC), Glorieta de la Astronomía s/n Aptdo. 3004, 18080 Granada, Spain

9 Observatorio Astronómico, Laprida 854, X5000BGR Córdoba, Argentina
${ }^{10}$ Consejo de Investigaciones Científicas y Técnicas de la República Argentina, Avda. Rivadavia 1917, C1033AAJ CABA, Argentina

11 INAF-Osservatorio Astrofisico di Arcetri - Largo Enrico Fermi, 50125 Firenze, Italy

12 Shanghai Astronomical Observatory, Chinese Academy of Sciences, 80 Nandan Road, Shanghai 200030, PR China

13 PITT PACC, Department of Physics and Astronomy, University of Pitts-burgh, Pittsburgh, PA 15260, USA

14 Departamento de Física Teórica, Universidad Autónoma de Madrid, 28049 Madrid, Spain

15 School of Physics and Astronomy, University of St Andrews, North Haugh, St Andrews KY16 9SS, UK

16 Sydney Institute for Astronomy, School of Physics A28, University of Sydney, Sydney, NSW 2006, Australia

17 ARC Centre of Excellence for All-sky Astrophysics in 3D (ASTRO3D), Australia

18 Departamento de Física, Universidade Federal de Santa Catarina, PO Box 476, 88040-900 Florianópolis, SC, Brazil

19 ETH Zürich, Institute for Astronomy, Wolfgang-Pauli-Str. 27, 8093 Zürich, Switzerland

20 Kapteyn Astronomical Institute, University of Groningen, Postbus 800, 9700 Groningen, The Netherlands

${ }^{21}$ Departamento de Física Teórica y del Cosmos, University of Granada, Facultad de Ciencias (Edificio Mecenas), 18071 Granada, Spain

22 Instituto Carlos I de Física Teórica y Computación, Spain

23 Instituto Nacional de Astrofísica, Óptica y Electrónica, Luis E. Erro 1, 72840 Tonantzintla, Puebla, Mexico 


\section{Appendix A: Deprojection of $\lambda_{\mathrm{Re}}$}

For an oblate galaxy, viewed at inclination $i$, we obtain

$\varepsilon_{90^{\circ}}=1-\frac{\sqrt{(1-\varepsilon)^{2}-\cos ^{2} i}}{\sin i}$,

as the deprojection of the observed ellipticity $\varepsilon$ to the intrinsic ellipticity $\varepsilon_{90^{\circ}}$ when the galaxy would be viewed edge-on $\left(i=90^{\circ}\right)$. Defining the global anisotropy parameter as $\delta \equiv$ $1-2 \sigma_{z}^{2} /\left(\sigma_{\mathrm{R}}^{2}+\sigma_{\phi}^{2}\right)$, we infer as shown by Binney (2005)

$(V / \sigma)_{90^{\circ}}=\frac{\sqrt{1-\delta \cos ^{2} i}}{\sin i}(V / \sigma)$,

for the deprojection of the observed ordered-over-random motion to its edge-on value. Next, inserting this into the approximate relation between $\lambda_{\mathrm{R}}$ and $V / \sigma$ (see Eq. (B1) of Emsellem et al. 2011), we find

$\lambda_{\mathrm{R}, 90^{\circ}} \simeq \frac{\sqrt{1-\delta \cos ^{2} i}}{\sin i} \frac{\lambda_{\mathrm{R}}}{\sqrt{1+(1-\delta) \cot ^{2} i \lambda_{\mathrm{R}}^{2}}}$,

as the approximate deprojection of the observed apparent stellar angular momentum $\lambda_{\mathrm{R}}$ to its edge-on value $\lambda_{\mathrm{R}, 90^{\circ}}$.

The inclination of a galaxy can be inferred directly from observations only in special cases, for example when a intrinsically thin and circular disk (in cold gas or corresponding dust) is present, so that its inclination follows directly from the observed ellipticity because $1-\varepsilon=\cos i$. In general, even if disks are close to axisymmetric, they have a non-negligible intrinsic flattening $q$ which, moreover, will vary from galaxy-to-galaxy.

If we assume that each galaxy is drawn from a group of galaxies with intrinsic shape distribution $f(q)$, the probability of viewing the galaxy at inclination $i$ is given by its observed ellipticity $\varepsilon$ as

$$
f(i \mid \varepsilon)=\frac{f(q)(1-\varepsilon)}{\sqrt{\sin ^{2} i-\varepsilon(2-\varepsilon)}},
$$

for $0 \leq \cos i<1-\varepsilon$, and zero otherwise.
We adopt for $f(q)$ a Gaussian distribution with mean and standard deviation $\left(\mu_{q}, \sigma_{q}\right)$, even though it is widely known that this cannot fit the observed ellipticity distribution of a complete (and hence randomly inclined) sample of galaxies. For example, Lambas et al. (1992) introduce even for spiral galaxies an additional Gaussian distribution in the intermediate-to-long axis ratio $p$ with mean around the oblate case of $p=1$, but also non-zero dispersion to fit the tail toward rounder galaxies. However, the effect on the inferred (median) inclination is small, even for the mildly triaxial slow-rotator elliptical galaxies, so that we assume an oblate intrinsic shape for all galaxies. Even more so, it turns out that the Gaussian distribution with $\left(\mu_{q}, \sigma_{q}\right)=(0.25,0.12)$ inferred for 13482 spirals by Lambas et al. (1992) is nearly identical to the Gaussian distribution with $\left(\mu_{q}, \sigma_{q}\right)=(0.25,0.14)$ inferred the fast-rotator E/S0 galaxies from the ATLAS ${ }^{3 \mathrm{D}}$ survey by Weijmans et al. (2014). Henceforth, for all fast-rotator galaxies we adopt the latter Gaussian intrinsic shape distribution, whereas Weijmans et al. (2014) find that the intrinsic shape distribution of the slow-rotator galaxies is well-described by a Gaussian with $\left(\mu_{q}, \sigma_{q}\right)=(0.63,0.09)$.

Based on axisymmetric dynamical models of $24 \mathrm{E} / \mathrm{S} 0$ galaxies, Cappellari et al. (2007) find that their velocity distribution is close to isotropic in the equatorial plane $\left(\sigma_{\mathrm{R}} \sim \sigma_{\phi}\right)$ and the remaining anisotropy in the meridional plane $(\delta \simeq \beta \equiv$ $1-\sigma_{z}^{2} / \sigma_{\mathrm{R}}^{2}$ ) is approximately linearly correlated with intrinsic ellipticity $\varepsilon_{90^{\circ}}$. Based on this correlation, adopting a Gaussian distribution in $\delta$ with mean $\mu_{\delta}=0.5 \varepsilon_{90^{\circ}}$ and standard deviation $\sigma_{\delta}=0.1$ for $0 \leq \delta \leq 0.8 \varepsilon_{90^{\circ}}$ and zero elsewhere, Emsellem et al. (2011) can explain the observed $(V / \sigma, \varepsilon)$-diagram of the complete sample of $\mathrm{ATLAS}^{3 \mathrm{D}}$ fast-rotator $\mathrm{E} / \mathrm{S} 0$ galaxies.

We followed the procedure above for each CALIFA galaxy to approximate the observed $\lambda_{\mathrm{Re}}$ values into intrinsic $\lambda_{\mathrm{Re}, 90^{\circ}}$ values at an edge-on view. First by adopting the above fast-rotator or slow-rotator Gaussian intrinsic shape distribution $f(q)$ to obtain the average inclination $i_{\text {av }}$ from the median of the corresponding distribution in inclinations given by Eq. (A.4). Second by inserting $i_{\text {av }}$ and the observed ellipticity in $\varepsilon$ into Eq. (A.1), which provides the approximate intrinsic ellipticity $\varepsilon_{90^{\circ}}$. Finally, adopting the above Gaussian distribution in the global anisotropy $\sigma_{\delta}$, Eq. (A.3) provides the approximate deprojection to the intrinsic stellar angular momentum $\lambda_{\mathrm{Re}, 90^{\circ}}$ within the effective radius $R_{\mathrm{e}}$. 
Table B.1. Stellar angular momentum properties of the CALIFA stellar kinematics sample.

\begin{tabular}{|c|c|c|c|c|c|c|c|c|c|c|c|c|c|c|c|}
\hline ID & $\epsilon$ & $\begin{array}{c}\text { PA } \\
\text { (deg) }\end{array}$ & $\begin{array}{l}\text { Incl. } \\
\text { (deg) }\end{array}$ & $\begin{array}{c}R_{\mathrm{eff}} \\
(\operatorname{arcsec})\end{array}$ & Type & $\begin{array}{c}M_{*} \\
\left(10^{10} M_{\odot}\right)\end{array}$ & $\begin{array}{c}M_{r} \\
\text { (mag) }\end{array}$ & $C_{90 / 50}$ & $\begin{array}{c}u-r \\
(\mathrm{mag})\end{array}$ & $\begin{array}{c}\mathrm{SFR} \\
\left(M_{\odot} \mathrm{yr}^{-1}\right)\end{array}$ & $\lambda_{0.5 \mathrm{Re}}$ & $\lambda_{\operatorname{Re}}$ & $\lambda_{2 \operatorname{Re}}$ & $\lambda_{\mathrm{Re}, 90^{\circ}}$ & $(V / \sigma)_{\mathrm{e}}$ \\
\hline 1 & 0.685 & 3.4 & 75.8 & 16 & $\mathrm{Sb}$ & 4.52 & -21.10 & 3.33 & 2.78 & 0.98 & 0.51 & 0.60 & 0.74 & 0.61 & 0.67 \\
\hline 2 & 0.526 & 44.1 & 65.8 & 16 & Sbc & 6.78 & -22.09 & 2.06 & 2.86 & 6.43 & 0.71 & 0.83 & - & 0.84 & 1.45 \\
\hline 3 & 0.412 & 105.3 & 56.8 & 23 & $\mathrm{Sc}$ & 2.45 & -21.06 & 2.11 & 2.44 & 2.18 & 0.52 & 0.61 & - & 0.64 & 0.74 \\
\hline 4 & 0.302 & 173.3 & 64.6 & 17 & $\mathrm{E} 1$ & 10.86 & -22.66 & 3.10 & 2.83 & 0.13 & 0.07 & 0.11 & - & 0.12 & 0.13 \\
\hline 5 & 0.559 & 75.1 & 67.8 & 23 & Sbc & 1.39 & -20.95 & 2.12 & 2.27 & 1.41 & 0.75 & 0.84 & - & 0.85 & 1.55 \\
\hline 6 & 0.370 & 143.3 & 53.6 & 11 & $\mathrm{Sab}$ & 17.62 & -22.26 & 3.22 & 3.29 & 0.72 & 0.39 & 0.50 & 0.64 & 0.54 & 0.53 \\
\hline 7 & 0.611 & 18.0 & 71.8 & 10 & $\mathrm{Sab}$ & 10.05 & -21.69 & 2.68 & 2.86 & 1.33 & 0.37 & 0.58 & - & 0.59 & 0.67 \\
\hline 8 & 0.324 & 107.6 & 50.0 & 12 & Sbc & 6.31 & -21.73 & 3.04 & 2.39 & 4.87 & 0.44 & 0.53 & 0.64 & 0.58 & 0.58 \\
\hline 9 & 0.302 & 177.5 & 48.1 & 17 & $\mathrm{Sb}$ & 10.96 & -22.47 & 3.22 & 2.24 & 15.50 & 0.45 & 0.43 & - & 0.48 & 0.48 \\
\hline 10 & 0.476 & 24.6 & 61.9 & 21 & $\mathrm{Sb}$ & 7.87 & -22.33 & 2.49 & 2.64 & 2.47 & 0.61 & 0.75 & - & 0.77 & 1.00 \\
\hline 12 & 0.755 & 96.8 & 79.8 & 20 & Sc & 1.29 & -20.75 & 2.22 & 1.91 & 3.09 & 0.64 & 0.73 & - & 0.73 & 0.98 \\
\hline 13 & 0.561 & 171.2 & 68.6 & 19 & $\mathrm{Sb}$ & 2.34 & -20.88 & 2.24 & 2.48 & 0.69 & 0.55 & 0.73 & - & 0.74 & 0.98 \\
\hline 14 & 0.462 & 7.5 & 60.9 & 20 & $\mathrm{Sd}$ & 0.60 & -20.69 & 2.08 & 1.79 & 3.69 & 0.41 & 0.51 & - & 0.53 & 0.54 \\
\hline 16 & 0.468 & 53.4 & 61.5 & 20 & Scd & 0.99 & -20.19 & 2.23 & 2.24 & 1.19 & 0.51 & 0.61 & - & 0.63 & 0.74 \\
\hline 17 & 0.490 & 149.8 & 63.2 & 18 & $\mathrm{E} 4$ & 6.07 & -21.39 & 3.23 & 3.58 & 0.10 & 0.25 & 0.28 & - & 0.29 & 0.29 \\
\hline 18 & 0.143 & 167.6 & 41.8 & 15 & E1 & 15.00 & -22.41 & 3.01 & 2.70 & 0.10 & 0.06 & 0.10 & - & 0.13 & 0.10 \\
\hline 20 & 0.473 & 49.8 & 61.6 & 22 & $\mathrm{Sa}$ & 10.72 & -22.18 & 2.98 & 3.02 & 0.34 & 0.33 & 0.45 & - & 0.47 & 0.43 \\
\hline 22 & 0.473 & 90.7 & 61.7 & 34 & $\mathrm{Sab}$ & 39.90 & -21.87 & 2.69 & 3.80 & 1.82 & 0.63 & 0.65 & - & 0.67 & 0.81 \\
\hline 23 & 0.055 & 32.0 & 20.7 & 26 & $\mathrm{Sb}$ & 5.26 & -21.84 & 1.98 & 2.64 & 0.69 & 0.29 & 0.53 & - & 0.80 & 0.47 \\
\hline 24 & 0.417 & 8.2 & 57.4 & 13 & $\mathrm{Sab}$ & 2.34 & -20.70 & 3.79 & 3.10 & - & 0.56 & 0.68 & 0.86 & 0.70 & 0.82 \\
\hline 25 & 0.339 & 167.1 & 51.1 & 28 & $\mathrm{Sb}$ & 8.36 & -22.31 & 2.18 & 3.01 & 2.35 & 0.64 & 0.80 & - & 0.83 & 1.18 \\
\hline 26 & 0.574 & 168.7 & 68.9 & 22 & $\mathrm{Sab}$ & 7.05 & -21.59 & 2.63 & 2.70 & 2.69 & 0.56 & 0.75 & - & 0.76 & 0.91 \\
\hline 27 & 0.711 & 25.8 & 77.3 & 20 & $\mathrm{Sd}$ & 0.19 & -18.99 & 2.45 & 1.63 & 0.26 & 0.34 & 0.52 & - & 0.52 & 0.54 \\
\hline 28 & 0.261 & 50.5 & 44.5 & 18 & Sbc & 6.65 & -22.16 & 2.44 & 2.44 & 4.87 & 0.62 & 0.71 & - & 0.77 & 1.12 \\
\hline 29 & 0.742 & 112.9 & 79.0 & 23 & $\mathrm{Sa}$ & 12.50 & -21.90 & 2.98 & 3.02 & - & 0.45 & 0.63 & - & 0.63 & 0.65 \\
\hline 30 & 0.324 & 175.3 & 50.0 & 15 & $\mathrm{Sc}$ & 2.04 & -21.14 & 2.42 & 1.88 & 2.18 & 0.50 & 0.63 & 0.66 & 0.68 & 0.82 \\
\hline 31 & 0.205 & 64.3 & 39.1 & 20 & $\mathrm{Sc}$ & 4.50 & -21.91 & 1.91 & 2.45 & 5.75 & 0.54 & 0.64 & - & 0.73 & 0.83 \\
\hline 32 & 0.743 & 22.7 & 79.0 & 12 & $\mathrm{Sab}$ & 4.24 & -21.41 & 3.13 & 2.64 & - & 0.55 & 0.67 & 0.82 & 0.68 & 0.75 \\
\hline 33 & 0.359 & 88.9 & 52.7 & 21 & $\mathrm{Sc}$ & 6.22 & -22.15 & 2.11 & 2.54 & 5.76 & 0.64 & 0.75 & - & 0.78 & 1.08 \\
\hline 35 & 0.275 & 35.4 & 45.7 & 15 & E7 & 9.16 & -21.56 & 3.10 & 3.06 & 0.04 & 0.43 & 0.57 & - & 0.63 & 0.60 \\
\hline 36 & 0.777 & 15.8 & 80.6 & 6 & $\mathrm{Sa}$ & 6.22 & -21.26 & 3.16 & 2.65 & 3.31 & 0.43 & 0.56 & 0.66 & 0.56 & 0.57 \\
\hline 37 & 0.724 & 171.5 & 78.3 & 11 & SOa & 4.09 & -21.20 & - & 2.58 & 0.22 & 0.39 & 0.58 & 0.73 & 0.58 & 0.62 \\
\hline 38 & 0.126 & 74.9 & 30.4 & 28 & $\mathrm{Sa}$ & 13.52 & -22.40 & 3.33 & 2.86 & 1.16 & 0.25 & 0.30 & - & 0.42 & 0.28 \\
\hline 39 & 0.739 & 158.7 & 79.1 & 23 & Scd & 0.74 & -20.23 & 2.25 & 2.09 & 0.67 & 0.62 & 0.76 & - & 0.76 & 1.12 \\
\hline 40 & 0.808 & 23.6 & 82.2 & 20 & Scd & 0.49 & -19.72 & 2.31 & 2.22 & 0.64 & 0.55 & 0.68 & - & 0.68 & 0.84 \\
\hline 41 & 0.774 & 54.5 & 80.4 & 17 & Sbc & 1.03 & -20.26 & 2.52 & 2.30 & 0.87 & 0.81 & 0.85 & - & 0.85 & 1.51 \\
\hline 42 & 0.500 & 128.4 & 64.0 & 21 & Sbc & 3.14 & -21.69 & - & 2.52 & 2.25 & 0.69 & 0.82 & - & 0.83 & 1.30 \\
\hline 43 & 0.355 & 15.6 & 52.7 & 13 & $\mathrm{Sb}$ & 3.88 & -21.11 & 2.59 & 2.66 & 3.17 & 0.50 & 0.64 & - & 0.68 & 0.74 \\
\hline 44 & 0.328 & 72.2 & 67.4 & 21 & E5 & 25.18 & -22.48 & 2.96 & 3.00 & 0.12 & 0.05 & 0.10 & - & 0.11 & 0.10 \\
\hline 45 & 0.455 & 32.7 & 60.2 & 19 & Scd & 2.59 & -21.40 & 2.03 & 2.49 & 4.14 & 0.78 & 0.83 & - & 0.84 & 1.53 \\
\hline 46 & 0.597 & 44.2 & 70.5 & 8 & S0 & 2.95 & -20.76 & 3.17 & 2.83 & 0.02 & 0.37 & 0.46 & 0.59 & 0.47 & 0.45 \\
\hline 47 & 0.485 & 24.2 & 62.6 & 10 & So & 6.64 & -21.35 & 3.46 & 2.79 & 0.02 & 0.43 & 0.50 & 0.52 & 0.51 & 0.51 \\
\hline 49 & 0.640 & 30.3 & 73.8 & 12 & $\mathrm{Sa}$ & 4.11 & -21.21 & 3.00 & 2.57 & 1.39 & 0.61 & 0.76 & 0.83 & 0.76 & 1.00 \\
\hline 50 & 0.516 & 57.7 & 65.1 & 12 & So & 7.48 & -21.68 & 3.06 & 3.03 & 0.46 & 0.53 & 0.66 & 0.74 & 0.67 & 0.72 \\
\hline 51 & 0.095 & 13.9 & 26.6 & 12 & E4 & 12.25 & -22.27 & 3.13 & 2.87 & 0.06 & 0.28 & 0.34 & 0.41 & 0.52 & 0.35 \\
\hline 52 & 0.564 & 137.5 & 68.2 & 19 & Sbc & 4.38 & -21.52 & 2.12 & 3.25 & 1.82 & 0.67 & 0.81 & 0.86 & 0.81 & 1.28 \\
\hline 53 & 0.686 & 152.4 & 75.9 & 14 & $\mathrm{Sc}$ & 1.27 & -20.81 & 2.44 & 2.10 & 2.17 & 0.62 & 0.72 & - & 0.72 & 0.97 \\
\hline 59 & 0.465 & 99.7 & 60.9 & 9 & SOa & 6.71 & -21.42 & 3.06 & 2.89 & 0.03 & 0.33 & 0.44 & 0.57 & 0.45 & 0.45 \\
\hline
\end{tabular}

Notes. Column 1: CALIFA ID. Column 2: average ellipticity measured in the outer parts of the galaxy, using SDSS images. Column 3: average position angle measured in the outer parts of the galaxy, using SDSS images. Column 4: statistical inclination (see Appendix A). Column 5: effective radii (in arcsec) of the galaxy, measured as described in Walcher et al. (2014). Column 6: Hubble type of the galaxy from Walcher et al. (2014). Column 7: total stellar mass of the galaxy, measured as described in Walcher et al. (2014). Column 8: total absolute magnitude in $r$-band from SDSS (Abazajian et al. 2009). Column 9: concentration index (ratio of Petrosian radius rad90 and rad50). Column 10: SDSS Petrosian $u-r$ color. Col. 11: star formation rate based on extinction corrected $\mathrm{H} \alpha$ measurements (Sánchez et al. 2017). Columns 12,13,14: $\lambda_{\mathrm{R}}$ measured on an elliptical aperture with semi-major axis $0.5 R_{\mathrm{e}}, R_{\mathrm{e}}$, and $2 R_{\mathrm{e}}$ respectively. Column 15: deprojected $\lambda_{\mathrm{Re}}\left(\lambda_{\mathrm{Re}, 90^{\circ}} \text {, see Appendix A). Column 16: }(V / \sigma)\right)_{\mathrm{e}}$ measured on an elliptical aperture with semi-major axis $R_{\mathrm{e}}$. We refer the reader to FLV17 for further properties of the galaxies not listed here. 
Table B.1. continued.

\begin{tabular}{|c|c|c|c|c|c|c|c|c|c|c|c|c|c|c|c|}
\hline ID & $\epsilon$ & $\begin{array}{c}\text { PA } \\
\text { (deg) }\end{array}$ & $\begin{array}{l}\text { Incl. } \\
\text { (deg) }\end{array}$ & $\begin{array}{c}R_{\text {eff }} \\
(\operatorname{arcsec})\end{array}$ & Type & $\begin{array}{c}M_{*} \\
\left(10^{10} M_{\odot}\right)\end{array}$ & $\begin{array}{c}M_{r} \\
(\mathrm{mag})\end{array}$ & $C_{90 / 50}$ & $\begin{array}{c}u-r \\
(\mathrm{mag}) \\
\end{array}$ & $\begin{array}{c}\mathrm{SFR} \\
\left(M_{\odot} \mathrm{yr}^{-1}\right)\end{array}$ & $\lambda_{0.5 \mathrm{Re}}$ & $\lambda_{\operatorname{Re}}$ & $\lambda_{2 \mathrm{Re}}$ & $\lambda_{\mathrm{Re}, 90^{\circ}}$ & $(V / \sigma)_{\mathrm{e}}$ \\
\hline 61 & 0.328 & 65.1 & 50.2 & 30 & $\mathrm{Sa}$ & 3.10 & -20.71 & 2.44 & 3.15 & 0.35 & 0.29 & 0.28 & - & 0.32 & 0.34 \\
\hline 68 & 0.223 & 85.2 & 54.0 & 35 & E1 & 32.89 & -23.47 & 2.80 & 3.05 & 0.20 & 0.07 & 0.07 & - & 0.08 & 0.07 \\
\hline 69 & 0.610 & 49.2 & 71.9 & 28 & Scd & 0.24 & -19.43 & 2.16 & 1.77 & 0.36 & 0.43 & 0.53 & - & 0.54 & 0.60 \\
\hline 70 & 0.748 & 155.0 & 79.4 & 11 & $\mathrm{Sb}$ & 8.43 & -21.69 & 2.97 & 2.97 & 0.55 & 0.52 & 0.67 & 0.84 & 0.67 & 0.77 \\
\hline 71 & 0.607 & 33.6 & 71.5 & 15 & $\mathrm{Sc}$ & 3.48 & -21.78 & 2.54 & 2.17 & 4.33 & 0.57 & 0.73 & 0.77 & 0.73 & 0.94 \\
\hline 72 & 0.177 & 164.2 & 36.2 & 12 & S0 & 8.39 & -21.55 & 3.01 & 2.80 & 0.13 & 0.40 & 0.52 & 0.55 & 0.64 & 0.58 \\
\hline 73 & 0.101 & 41.3 & 27.2 & 19 & $\mathrm{Sb}$ & 4.94 & -21.82 & 2.10 & 2.90 & 3.19 & 0.24 & 0.37 & - & 0.54 & 0.34 \\
\hline 74 & 0.702 & 11.9 & 76.9 & 8 & $\mathrm{Sa}$ & 2.96 & -20.80 & 3.38 & 2.55 & 0.61 & 0.36 & 0.51 & 0.69 & 0.52 & 0.49 \\
\hline 76 & 0.344 & 27.3 & 69.0 & 17 & E5 & 35.65 & -22.84 & 2.98 & 3.06 & 0.30 & 0.10 & 0.14 & - & 0.15 & 0.16 \\
\hline 77 & 0.507 & 50.6 & 64.3 & 12 & $\mathrm{Sa}$ & 2.64 & -20.70 & 3.18 & 2.68 & 0.12 & 0.22 & 0.25 & 0.33 & 0.25 & 0.28 \\
\hline 87 & 0.084 & 65.3 & 24.7 & 18 & SOa & 9.20 & -22.10 & 3.20 & 3.14 & 0.36 & 0.24 & 0.31 & - & 0.50 & 0.29 \\
\hline 100 & 0.318 & 153.8 & 49.4 & 14 & $\mathrm{Sa}$ & 1.05 & -19.94 & 3.17 & 2.85 & 1.09 & 0.22 & 0.22 & 0.24 & 0.25 & 0.28 \\
\hline 101 & 0.180 & 75.0 & 47.5 & 27 & E3 & 70.15 & -23.62 & 3.03 & 3.52 & 0.24 & 0.04 & 0.05 & - & 0.06 & 0.06 \\
\hline 103 & 0.575 & 96.8 & 69.0 & 10 & SOa & 5.53 & -21.42 & 3.10 & 3.00 & 0.10 & 0.42 & 0.53 & 0.68 & 0.54 & 0.54 \\
\hline 104 & 0.521 & 177.2 & 65.4 & 19 & SOa & 7.76 & -22.03 & 3.18 & 2.73 & 0.35 & 0.57 & 0.58 & - & 0.59 & 0.68 \\
\hline 108 & 0.390 & 99.1 & 55.2 & 13 & Sbc & 3.25 & -21.49 & 2.61 & 2.70 & 1.18 & 0.59 & 0.71 & 0.77 & 0.74 & 0.98 \\
\hline 115 & 0.587 & 153.5 & 69.8 & 19 & $\mathrm{Sb}$ & 3.19 & -20.80 & 2.04 & 2.84 & 3.10 & 0.56 & 0.70 & - & 0.71 & 1.01 \\
\hline 119 & 0.228 & 62.5 & 41.3 & 24 & So & 49.20 & -22.98 & 2.75 & 3.77 & 1.33 & 0.32 & 0.44 & - & 0.52 & 0.44 \\
\hline 127 & 0.119 & 50.6 & 29.6 & 17 & E6 & 8.47 & -22.44 & 2.59 & 3.28 & 0.65 & 0.25 & 0.38 & - & 0.54 & 0.37 \\
\hline 131 & 0.589 & 131.3 & 70.1 & 15 & $\mathrm{Sab}$ & 2.74 & -20.74 & 2.52 & 3.05 & 0.72 & 0.48 & 0.62 & - & 0.63 & 0.73 \\
\hline 134 & 0.568 & 84.4 & 68.7 & 13 & SOa & 6.78 & -21.81 & 3.23 & 2.77 & 0.35 & 0.44 & 0.54 & 0.75 & 0.55 & 0.57 \\
\hline 135 & 0.725 & 93.5 & 78.5 & 20 & $\mathrm{Sa}$ & 5.71 & -21.41 & 2.43 & 10.64 & 0.82 & 0.56 & 0.77 & - & 0.77 & 1.30 \\
\hline 143 & 0.710 & 137.0 & 77.7 & 12 & Scd & 0.38 & -19.46 & 2.51 & 1.69 & 0.63 & 0.44 & 0.56 & 0.60 & 0.57 & 0.65 \\
\hline 144 & 0.724 & 144.2 & 78.0 & 25 & Scd & 1.74 & -20.81 & 2.48 & 2.11 & 1.86 & 0.67 & 0.76 & - & 0.76 & 1.03 \\
\hline 146 & 0.475 & 87.7 & 61.7 & 15 & $\mathrm{Sb}$ & 2.69 & -21.16 & 2.26 & 2.54 & - & 0.52 & 0.70 & - & 0.71 & 0.89 \\
\hline 147 & 0.323 & 109.7 & 49.8 & 15 & Sbc & 3.34 & -21.55 & 2.14 & 2.54 & 2.81 & 0.46 & 0.65 & 0.75 & 0.70 & 0.80 \\
\hline 148 & 0.693 & 117.9 & 76.8 & 20 & $\mathrm{Sc}$ & 0.70 & -19.69 & 2.82 & 2.50 & 0.21 & 0.63 & 0.74 & - & 0.75 & 1.03 \\
\hline 149 & 0.360 & 9.1 & 52.8 & 18 & Sbc & 8.71 & -22.12 & 2.57 & 2.34 & 2.65 & 0.76 & 0.79 & 0.82 & 0.82 & 1.29 \\
\hline 150 & 0.698 & 44.0 & 77.1 & 9 & $\mathrm{Sd}$ & 0.17 & -19.23 & 2.89 & 1.36 & 0.50 & 0.33 & 0.34 & 0.35 & 0.34 & 0.37 \\
\hline 151 & 0.684 & 34.6 & 76.1 & 21 & $\mathrm{Sb}$ & 7.62 & -21.86 & 3.09 & 2.62 & 2.66 & 0.63 & 0.73 & - & 0.73 & 0.90 \\
\hline 152 & 0.569 & 120.9 & 68.6 & 17 & $\mathrm{Sbc}$ & 0.99 & -20.42 & 2.16 & 2.01 & 0.69 & 0.66 & 0.71 & - & 0.71 & 1.01 \\
\hline 153 & 0.781 & 134.3 & 80.7 & 15 & $\mathrm{Sb}$ & 4.78 & -21.19 & 2.36 & 2.47 & 2.24 & 0.60 & 0.79 & - & 0.79 & 1.18 \\
\hline 155 & 0.555 & 90.7 & 67.7 & 25 & $\mathrm{Sb}$ & 8.36 & -22.12 & 2.43 & 3.45 & 1.31 & 0.54 & 0.65 & - & 0.66 & 0.75 \\
\hline 156 & 0.519 & 135.7 & 65.4 & 16 & $\mathrm{Sab}$ & 7.28 & -21.68 & 2.74 & 2.97 & 0.94 & 0.62 & 0.76 & - & 0.77 & 1.03 \\
\hline 171 & 0.269 & 174.5 & 60.4 & 26 & $\mathrm{E} 2$ & 34.59 & -22.86 & 3.08 & 3.14 & 0.10 & 0.08 & 0.10 & - & 0.11 & 0.10 \\
\hline 174 & 0.790 & 130.7 & 81.2 & 18 & $\mathrm{Sab}$ & 5.15 & -20.92 & - & 3.00 & 0.66 & 0.71 & 0.81 & 0.89 & 0.81 & 1.20 \\
\hline 183 & 0.394 & 131.5 & 55.6 & 14 & Sbc & 3.32 & -21.62 & 2.17 & 2.08 & 2.91 & 0.65 & 0.78 & 0.83 & 0.80 & 1.20 \\
\hline 185 & 0.682 & 3.7 & 75.9 & 11 & $\mathrm{Sb}$ & 1.37 & -20.29 & 2.58 & 2.43 & 0.48 & 0.53 & 0.69 & 0.83 & 0.70 & 0.86 \\
\hline 186 & 0.787 & 148.5 & 81.4 & 21 & Sab & 3.24 & -20.75 & - & 3.29 & 1.20 & 0.63 & 0.70 & - & 0.70 & 0.88 \\
\hline 187 & 0.141 & 113.9 & 32.8 & 24 & $\mathrm{Sc}$ & 1.84 & -21.29 & 2.06 & 3.96 & 1.11 & 0.50 & 0.68 & - & 0.80 & 0.94 \\
\hline 188 & 0.499 & 67.6 & 63.7 & 9 & $\mathrm{Sb}$ & 6.89 & -21.30 & 3.22 & 2.76 & 0.03 & 0.41 & 0.49 & - & 0.51 & 0.52 \\
\hline 189 & 0.192 & 160.4 & 37.9 & 19 & SOa & 16.33 & -22.59 & 2.97 & 2.99 & 0.50 & 0.29 & 0.36 & - & 0.44 & 0.36 \\
\hline 201 & 0.217 & 45.9 & 40.2 & 9 & $\mathrm{E} 4$ & 4.15 & -20.72 & 3.25 & 2.81 & 0.01 & 0.36 & 0.44 & 0.56 & 0.52 & 0.43 \\
\hline 209 & 0.118 & 48.4 & 29.4 & 26 & $\mathrm{Sd}$ & 0.46 & -20.24 & 1.98 & 1.95 & 0.57 & 0.21 & 0.29 & - & 0.42 & 0.34 \\
\hline 219 & 0.350 & 130.3 & 52.2 & 17 & $\mathrm{Sa}$ & 14.72 & -22.33 & 2.71 & 2.77 & 3.58 & 0.50 & 0.65 & 0.72 & 0.69 & 0.81 \\
\hline 231 & 0.793 & 31.7 & 81.2 & 32 & $\mathrm{Sdm}$ & 0.05 & -18.18 & 2.13 & 3.15 & 0.09 & 0.48 & 0.45 & - & 0.45 & 0.48 \\
\hline 232 & 0.115 & 80.8 & 29.2 & 24 & Scd & 1.31 & -20.94 & 1.88 & 1.95 & 1.46 & 0.48 & 0.56 & - & 0.72 & 0.72 \\
\hline 272 & 0.356 & 142.9 & 52.7 & 18 & E7 & 4.69 & -21.10 & 3.26 & 2.73 & 0.01 & 0.42 & 0.53 & - & 0.57 & 0.55 \\
\hline 273 & 0.791 & 162.9 & 81.2 & 25 & $\mathrm{Sc}$ & 2.48 & -21.05 & 2.51 & 3.22 & 1.43 & 0.79 & 0.84 & - & 0.85 & 1.42 \\
\hline 274 & 0.630 & 170.0 & 72.8 & 14 & $\mathrm{Sab}$ & 0.75 & -19.27 & 2.67 & 2.74 & 0.08 & 0.50 & 0.65 & - & 0.66 & 0.79 \\
\hline 275 & 0.437 & 82.6 & 59.1 & 19 & Sbc & 2.46 & -20.79 & 2.08 & 3.07 & 0.84 & 0.59 & 0.76 & - & 0.78 & 1.04 \\
\hline 277 & 0.356 & 19.9 & 52.3 & 26 & Sbc & 5.66 & -22.09 & 2.16 & 2.24 & 1.35 & 0.59 & 0.77 & - & 0.80 & 1.12 \\
\hline 278 & 0.595 & 138.1 & 70.3 & 9 & $\mathrm{Sb}$ & 7.74 & -22.12 & 2.81 & 2.48 & 4.87 & 0.32 & 0.47 & - & 0.47 & 0.54 \\
\hline 279 & 0.307 & 75.1 & 48.5 & 12 & E6 & 27.73 & -22.78 & 3.01 & 2.80 & 0.16 & 0.26 & 0.30 & 0.31 & 0.34 & 0.32 \\
\hline 281 & 0.738 & 41.9 & 78.7 & 8 & SOa & 12.62 & -21.76 & 3.44 & 2.93 & 0.12 & 0.44 & 0.52 & 0.71 & 0.53 & 0.53 \\
\hline 311 & 0.102 & 116.1 & 27.6 & 21 & $\mathrm{Sab}$ & 16.29 & -22.79 & 3.06 & 3.02 & 0.92 & 0.20 & 0.25 & - & 0.39 & 0.23 \\
\hline 312 & 0.269 & 23.2 & 44.9 & 32 & $\mathrm{Sdm}$ & 0.12 & -19.17 & 1.90 & 2.03 & 0.22 & 0.31 & 0.43 & - & 0.49 & 0.51 \\
\hline 314 & 0.785 & 61.4 & 81.2 & 13 & $\mathrm{Sa}$ & 6.38 & -21.36 & 3.10 & 2.69 & 1.11 & 0.58 & 0.68 & 0.81 & 0.68 & 0.81 \\
\hline
\end{tabular}


Table B.1. continued.

\begin{tabular}{|c|c|c|c|c|c|c|c|c|c|c|c|c|c|c|c|}
\hline ID & $\epsilon$ & $\begin{array}{c}\text { PA } \\
(\mathrm{deg})\end{array}$ & $\begin{array}{l}\text { Incl. } \\
\text { (deg) }\end{array}$ & $\begin{array}{c}R_{\mathrm{eff}} \\
(\operatorname{arcsec})\end{array}$ & Type & $\begin{array}{c}M_{*} \\
\left(10^{10} M_{\odot}\right)\end{array}$ & $\begin{array}{c}M_{r} \\
(\mathrm{mag})\end{array}$ & $C_{90 / 50}$ & $\begin{array}{c}u-r \\
(\mathrm{mag})\end{array}$ & $\begin{array}{c}\mathrm{SFR} \\
\left(M_{\odot} \mathrm{yr}^{-1}\right)\end{array}$ & $\lambda_{0.5 \mathrm{Re}}$ & $\lambda_{\operatorname{Re}}$ & $\lambda_{2 \operatorname{Re}}$ & $\lambda_{\operatorname{Re}, 90^{\circ}}$ & $(V / \sigma)_{\mathrm{e}}$ \\
\hline 318 & 0.188 & 165.7 & 37.5 & 32 & E3 & 54.70 & -23.70 & 3.11 & 3.14 & 0.32 & 0.25 & 0.26 & - & 0.34 & 0.29 \\
\hline 319 & 0.756 & 140.8 & 79.6 & 15 & $\mathrm{Sab}$ & 8.99 & -21.51 & 2.71 & 2.79 & 0.79 & 0.49 & 0.65 & 0.81 & 0.65 & 0.70 \\
\hline 326 & 0.738 & 35.6 & 79.2 & 15 & $\mathrm{Sb}$ & 1.71 & -20.75 & 2.46 & 2.23 & 1.32 & 0.47 & 0.68 & - & 0.69 & 0.87 \\
\hline 339 & 0.459 & 173.3 & 60.5 & 13 & SOa & 5.78 & -21.41 & 2.69 & 2.92 & 0.02 & 0.41 & 0.56 & 0.76 & 0.59 & 0.62 \\
\hline 340 & 0.508 & 159.6 & 79.1 & 15 & SOa & 11.51 & -22.33 & 2.97 & 2.76 & 1.86 & 0.20 & 0.19 & - & 0.19 & 0.21 \\
\hline 341 & 0.329 & 60.4 & 50.6 & 12 & E6 & 20.75 & -22.35 & 3.35 & 2.76 & 0.86 & 0.39 & 0.48 & 0.54 & 0.52 & 0.49 \\
\hline 353 & 0.141 & 43.1 & 32.4 & 24 & $\mathrm{Sd}$ & 0.48 & -20.08 & 2.03 & 1.89 & 0.30 & 0.21 & 0.25 & - & 0.35 & 0.29 \\
\hline 361 & 0.742 & 15.1 & 78.9 & 12 & $\mathrm{Sc}$ & 0.16 & -18.32 & 2.78 & 1.90 & - & 0.16 & 0.17 & 0.34 & 0.17 & 0.20 \\
\hline 364 & 0.726 & 100.6 & 78.6 & 11 & $\mathrm{Sa}$ & 14.86 & -21.93 & 3.41 & 2.79 & 0.50 & 0.43 & 0.56 & 0.80 & 0.57 & 0.55 \\
\hline 381 & 0.635 & 117.0 & 73.1 & 9 & $\mathrm{Sab}$ & 8.09 & -22.07 & 2.91 & 2.60 & 0.86 & 0.54 & 0.61 & 0.77 & 0.62 & 0.70 \\
\hline 386 & 0.636 & 49.6 & 73.3 & 13 & $\mathrm{Sab}$ & 10.74 & -21.91 & 3.00 & 2.77 & 0.83 & 0.40 & 0.50 & 0.63 & 0.51 & 0.51 \\
\hline 387 & 0.416 & 42.9 & 57.6 & 15 & E5 & 24.15 & -22.98 & 3.34 & 2.94 & 0.12 & 0.31 & 0.35 & - & 0.37 & 0.35 \\
\hline 414 & 0.058 & 151.0 & 20.5 & 17 & $\mathrm{Sb}$ & 1.88 & -20.97 & 2.21 & 2.46 & 0.42 & 0.36 & 0.52 & - & 0.77 & 0.56 \\
\hline 436 & 0.233 & 171.5 & 41.7 & 21 & Sbc & 2.65 & -21.40 & 2.14 & 2.34 & 2.00 & 0.45 & 0.64 & - & 0.71 & 0.76 \\
\hline 437 & 0.499 & 67.8 & 63.8 & 14 & Sbc & 2.25 & -21.05 & 2.65 & 2.12 & - & 0.57 & 0.66 & 0.65 & 0.67 & 0.88 \\
\hline 476 & 0.492 & 8.1 & 63.5 & 9 & Sbc & 2.65 & -21.22 & 2.72 & 1.89 & 3.71 & 0.54 & 0.67 & 0.71 & 0.69 & 0.84 \\
\hline 479 & 0.279 & 168.8 & 46.1 & 14 & SOa & 11.83 & -22.00 & 2.77 & 2.75 & 1.32 & 0.49 & 0.60 & - & 0.66 & 0.72 \\
\hline 486 & 0.507 & 12.1 & 64.4 & 14 & Scd & 0.28 & -19.91 & 2.72 & 1.48 & 0.69 & 0.62 & 0.71 & 0.63 & 0.73 & 1.00 \\
\hline 489 & 0.258 & 97.6 & 44.0 & 16 & Sbc & 4.86 & -21.90 & 2.29 & 2.17 & 3.42 & 0.61 & 0.66 & - & 0.73 & 0.94 \\
\hline 500 & 0.632 & 151.3 & 72.9 & 16 & Sbc & 2.26 & -21.14 & 2.31 & 2.43 & 1.23 & 0.71 & 0.83 & - & 0.83 & 1.40 \\
\hline 502 & 0.597 & 85.4 & 70.7 & 18 & $\mathrm{Sa}$ & 2.30 & -20.63 & 2.62 & 2.30 & 0.42 & 0.56 & 0.72 & - & 0.73 & 0.93 \\
\hline 515 & 0.334 & 164.4 & 50.7 & 30 & $\mathrm{Sbc}$ & 4.69 & -21.88 & 1.96 & 3.16 & 0.89 & 0.75 & 0.80 & - & 0.83 & 1.34 \\
\hline 518 & 0.244 & 97.7 & 42.7 & 21 & $\mathrm{Sb}$ & 1.93 & -20.98 & 1.87 & 2.55 & 0.55 & 0.64 & 0.79 & - & 0.84 & 1.25 \\
\hline 548 & 0.325 & 179.5 & 49.9 & 15 & $\mathrm{Sc}$ & 0.98 & -20.72 & 2.01 & 1.56 & 0.87 & 0.37 & 0.50 & - & 0.55 & 0.59 \\
\hline 569 & 0.706 & 57.0 & 77.3 & 12 & $\mathrm{Sb}$ & 2.82 & -21.03 & 2.78 & 2.40 & 0.80 & 0.58 & 0.73 & 0.83 & 0.74 & 0.96 \\
\hline 577 & 0.847 & 2.3 & 83.5 & 38 & Sdm & 6.50 & -22.17 & 2.80 & 2.41 & 2.09 & 0.49 & 0.54 & - & 0.54 & 0.62 \\
\hline 580 & 0.475 & 41.4 & 62.0 & 17 & $\mathrm{Sbc}$ & 2.05 & -21.05 & 2.04 & 2.38 & - & 0.73 & 0.76 & - & 0.78 & 1.19 \\
\hline 588 & 0.309 & 80.6 & 65.4 & 30 & E1 & 32.06 & -23.03 & 2.87 & 2.91 & 0.18 & 0.07 & 0.08 & - & 0.08 & 0.08 \\
\hline 589 & 0.107 & 42.9 & 28.0 & 20 & E3 & 35.16 & -22.83 & 3.22 & 2.84 & 0.21 & 0.08 & 0.10 & - & 0.17 & 0.12 \\
\hline 592 & 0.235 & 46.5 & 55.7 & 55 & E0 & 49.54 & -24.11 & 2.72 & 3.00 & 0.13 & 0.05 & 0.06 & - & 0.07 & 0.07 \\
\hline 593 & 0.681 & 53.2 & 75.8 & 16 & $\mathrm{Sa}$ & 11.64 & -22.56 & 2.49 & 3.17 & 6.40 & 0.34 & 0.44 & - & 0.45 & 0.44 \\
\hline 602 & 0.166 & 39.6 & 35.0 & 9 & E1 & 9.68 & -22.38 & 2.97 & 2.53 & 0.11 & 0.30 & 0.38 & 0.46 & 0.48 & 0.40 \\
\hline 603 & 0.313 & 100.6 & 48.8 & 15 & Scd & 0.48 & -20.25 & 2.46 & 1.59 & 0.64 & 0.45 & 0.51 & 0.48 & 0.56 & 0.61 \\
\hline 606 & 0.664 & 73.6 & 74.9 & 19 & $\mathrm{Sd}$ & 0.14 & -19.28 & 2.25 & 1.58 & 0.39 & 0.37 & 0.40 & - & 0.41 & 0.40 \\
\hline 607 & 0.454 & 133.4 & 60.5 & 8 & So & 13.65 & -22.76 & 3.32 & 2.39 & 0.14 & 0.40 & 0.51 & 0.59 & 0.53 & 0.54 \\
\hline 608 & 0.241 & 1.3 & 42.9 & 16 & Sbc & & -21.81 & 2.19 & 2.42 & 2.04 & 0.28 & 0.36 & - & 0.43 & 0.37 \\
\hline 611 & 0.233 & 57.4 & 41.7 & 17 & Sbc & 1.72 & -21.06 & 2.28 & 1.90 & 0.90 & 0.51 & 0.65 & - & 0.73 & 0.89 \\
\hline 612 & 0.397 & 149.2 & 73.2 & 25 & E6 & 31.77 & -23.28 & 3.02 & 2.88 & 0.17 & 0.07 & 0.09 & - & 0.09 & 0.10 \\
\hline 614 & 0.436 & 3.4 & 58.7 & 15 & $\mathrm{Sc}$ & 3.02 & -21.82 & 2.46 & 2.10 & - & 0.71 & 0.80 & 0.83 & 0.82 & 1.30 \\
\hline 630 & 0.354 & 169.0 & 52.2 & 19 & Sbc & 0.73 & -20.12 & 2.42 & 2.43 & 0.10 & 0.51 & 0.66 & 0.72 & 0.70 & 0.85 \\
\hline 633 & 0.325 & 33.2 & 50.0 & 20 & E0 & 3.20 & -21.07 & 2.95 & 2.71 & 0.08 & 0.19 & 0.20 & - & 0.23 & 0.22 \\
\hline 634 & 0.135 & 101.4 & 33.1 & 18 & $\mathrm{Sab}$ & 4.49 & -21.43 & 2.48 & 2.85 & 2.30 & 0.35 & 0.45 & - & 0.58 & 0.52 \\
\hline 657 & 0.437 & 21.5 & 59.2 & 30 & Sdm & 0.26 & -19.75 & 2.14 & 1.67 & 0.19 & 0.45 & 0.46 & - & 0.48 & 0.53 \\
\hline 663 & 0.649 & 105.7 & 74.3 & 19 & $\mathrm{Sab}$ & 18.20 & -22.37 & 2.76 & 3.07 & 2.08 & 0.57 & 0.70 & - & 0.71 & 0.83 \\
\hline 664 & 0.704 & 116.6 & 77.0 & 15 & $\mathrm{Sb}$ & 1.76 & -20.30 & 2.41 & 2.41 & 0.22 & 0.52 & 0.71 & - & 0.72 & 0.89 \\
\hline 665 & 0.403 & 160.7 & 56.3 & 15 & $\mathrm{Sb}$ & 11.38 & -22.37 & 2.47 & 2.77 & 1.51 & 0.41 & 0.62 & - & 0.65 & 0.67 \\
\hline 676 & 0.216 & 86.7 & 40.3 & 24 & $\mathrm{Sb}$ & 3.83 & -21.27 & 2.42 & 2.88 & 0.10 & 0.42 & 0.58 & - & 0.66 & 0.60 \\
\hline 684 & 0.293 & 111.4 & 47.2 & 20 & $\mathrm{Sb}$ & 18.75 & -22.57 & 2.31 & 2.83 & 1.72 & 0.52 & 0.72 & - & 0.76 & 0.88 \\
\hline 707 & 0.182 & 41.9 & 37.3 & 25 & Scd & 1.38 & -20.76 & 1.93 & 1.87 & 1.21 & 0.48 & 0.61 & - & 0.72 & 0.78 \\
\hline 708 & 0.321 & 174.5 & 49.7 & 31 & E5 & 10.57 & -21.95 & 2.79 & 2.82 & 0.02 & 0.15 & 0.19 & - & 0.21 & 0.20 \\
\hline 714 & 0.537 & 12.6 & 66.4 & 14 & Sbc & 3.82 & -21.85 & 2.39 & 1.83 & 4.47 & 0.73 & 0.82 & - & 0.83 & 1.41 \\
\hline 715 & 0.486 & 63.1 & 62.9 & 12 & Sbc & 0.73 & -20.18 & 3.03 & 2.28 & 0.82 & 0.53 & 0.64 & 0.79 & 0.66 & 0.80 \\
\hline 740 & 0.190 & 128.2 & 37.5 & 18 & $\mathrm{Sa}$ & 19.86 & -22.77 & 3.05 & 2.83 & 1.89 & 0.17 & 0.19 & - & 0.25 & 0.20 \\
\hline 744 & 0.058 & 30.7 & 20.6 & 19 & SO & 8.47 & -21.74 & 3.21 & 2.72 & 0.07 & 0.14 & 0.11 & - & 0.22 & 0.15 \\
\hline 748 & 0.256 & 16.9 & 43.9 & 13 & Sbc & 1.82 & -20.94 & 2.15 & 1.84 & 1.39 & 0.51 & 0.64 & 0.68 & 0.71 & 0.87 \\
\hline 749 & 0.705 & 93.7 & 77.1 & 22 & $\mathrm{Sdm}$ & 0.47 & -20.37 & 2.51 & 1.51 & 0.93 & 0.50 & 0.62 & - & 0.62 & 0.75 \\
\hline 754 & 0.630 & 164.0 & 72.4 & 10 & $\mathrm{Sbc}$ & 1.92 & -20.98 & 3.06 & 2.35 & 1.65 & 0.56 & 0.63 & 0.73 & 0.64 & 0.75 \\
\hline 758 & 0.764 & 125.6 & 80.3 & 26 & Scd & 0.25 & -19.39 & - & 1.75 & 0.26 & 0.62 & 0.68 & - & 0.68 & 0.83 \\
\hline
\end{tabular}


Table B.1. continued.

\begin{tabular}{|c|c|c|c|c|c|c|c|c|c|c|c|c|c|c|c|}
\hline ID & $\epsilon$ & $\begin{array}{c}\text { PA } \\
\text { (deg) }\end{array}$ & $\begin{array}{l}\text { Incl. } \\
\text { (deg) }\end{array}$ & $\begin{array}{c}R_{\text {eff }} \\
(\operatorname{arcsec})\end{array}$ & Type & $\begin{array}{c}M_{*} \\
\left(10^{10} M_{\odot}\right)\end{array}$ & $\begin{array}{c}M_{r} \\
(\mathrm{mag})\end{array}$ & $C_{90 / 50}$ & $\begin{array}{c}u-r \\
(\mathrm{mag}) \\
\end{array}$ & $\begin{array}{c}\mathrm{SFR} \\
\left(M_{\odot} \mathrm{yr}^{-1}\right)\end{array}$ & $\lambda_{0.5 \mathrm{Re}}$ & $\lambda_{\operatorname{Re}}$ & $\lambda_{2 \mathrm{Re}}$ & $\lambda_{\mathrm{Re}, 90^{\circ}}$ & $(V / \sigma)_{\mathrm{e}}$ \\
\hline 764 & 0.435 & 131.0 & 58.7 & 16 & Sbc & 7.05 & -22.29 & 2.36 & 2.44 & 1.78 & 0.52 & 0.71 & - & 0.74 & 0.86 \\
\hline 768 & .476 & 43.2 & 62.1 & 14 & Sbc & 0.85 & -20.46 & 2.30 & 1.94 & 0.86 & 0.69 & 0.73 & 0.74 & 0.74 & 1.01 \\
\hline 769 & 0.337 & 132.0 & 51.0 & 21 & Sbc & 1.61 & -20.95 & 1.84 & 2.13 & 0.97 & 0.61 & 0.72 & - & 0.76 & 1.06 \\
\hline 774 & 0.790 & 140.7 & 81.2 & 20 & $\mathrm{Sb}$ & 16.60 & -22.64 & 3.33 & 3.17 & 2.22 & 0.60 & 0.71 & - & 0.71 & 0.82 \\
\hline 775 & 0.703 & 34.3 & 77.1 & 21 & $\mathrm{Sc}$ & 2.07 & -20.96 & 2.29 & 2.38 & 1.67 & 0.73 & 0.78 & - & 0.78 & 1.18 \\
\hline 778 & 0.134 & 19.2 & 31.4 & 13 & S0 & 16.44 & -22.61 & 3.36 & 2.90 & 1.28 & 0.25 & 0.33 & 0.40 & 0.46 & 0.36 \\
\hline 780 & 0.453 & 130.1 & 60.1 & 18 & E7 & 7.01 & -22.13 & 3.14 & 2.54 & 0.04 & 0.43 & 0.51 & - & 0.53 & 0.53 \\
\hline 781 & 0.511 & 82.0 & 78.6 & 37 & E4 & 21.23 & -23.21 & 2.95 & 2.91 & 0.15 & 0.08 & 0.08 & - & 0.08 & 0.09 \\
\hline 783 & 0.725 & 138.2 & 78.3 & 18 & $\mathrm{Sb}$ & 0.99 & -19.99 & 2.37 & 2.47 & 0.80 & 0.63 & 0.76 & - & 0.76 & 1.10 \\
\hline 787 & 0.592 & 51.4 & 70.3 & 12 & SOa & 7.96 & -21.41 & 3.68 & 2.79 & 0.11 & 0.29 & 0.35 & 0.59 & 0.36 & 0.33 \\
\hline 789 & 0.382 & 150.7 & 54.8 & 16 & $\mathrm{Sb}$ & 16.07 & -22.74 & 2.07 & 2.87 & 2.30 & 0.57 & 0.76 & - & 0.79 & 1.03 \\
\hline 791 & 0.359 & 154.0 & 52.8 & 34 & $\mathrm{Sa}$ & 16.71 & -22.17 & 2.69 & 3.80 & 2.63 & 0.60 & 0.71 & - & 0.75 & 0.99 \\
\hline 795 & 0.537 & 161.8 & 66.5 & 16 & $\mathrm{Sab}$ & 4.30 & -21.36 & 2.77 & 2.70 & 2.23 & 0.59 & 0.72 & 0.78 & 0.73 & 0.92 \\
\hline 796 & 0.591 & 24.1 & 70.4 & 13 & $\mathrm{Sb}$ & 8.75 & -21.80 & 2.29 & 2.65 & 1.24 & 0.52 & 0.66 & 0.76 & 0.66 & 0.81 \\
\hline 797 & 0.746 & 126.0 & 79.2 & 21 & $\mathrm{Sb}$ & 1.25 & -20.38 & 2.44 & 2.51 & 1.19 & 0.56 & 0.71 & - & 0.71 & 0.90 \\
\hline 798 & 0.695 & 101.0 & 76.7 & 16 & Sbc & 1.98 & -20.71 & 2.50 & 2.29 & 0.89 & 0.64 & 0.80 & - & 0.80 & 1.22 \\
\hline 801 & 0.102 & 43.3 & 27.4 & 10 & $\mathrm{Sa}$ & 3.01 & -21.09 & 3.12 & 2.15 & 2.59 & 0.31 & 0.24 & 0.23 & 0.38 & 0.32 \\
\hline 804 & 0.555 & 132.4 & 68.0 & 12 & $\mathrm{Sb}$ & 2.07 & -20.80 & 2.96 & 2.28 & 0.12 & 0.38 & 0.51 & 0.62 & 0.52 & 0.53 \\
\hline 806 & 0.386 & 83.9 & 54.8 & 18 & E4 & 10.21 & -22.08 & 2.83 & 2.83 & 0.10 & 0.21 & 0.25 & - & 0.27 & 0.25 \\
\hline 807 & 0.411 & 161.0 & 57.1 & 15 & $\mathrm{Sb}$ & 8.99 & -21.96 & 2.24 & 2.95 & 0.70 & 0.49 & 0.62 & - & 0.65 & 0.71 \\
\hline 809 & 0.655 & 62.7 & 74.7 & 33 & $\mathrm{Sa}$ & 16.22 & -22.15 & 3.01 & 3.08 & 0.10 & 0.44 & 0.57 & - & 0.57 & 0.55 \\
\hline 810 & 0.596 & 11.7 & 70.2 & 17 & Sbc & 5.25 & -21.81 & 2.32 & 2.38 & 5.71 & 0.70 & 0.79 & 0.85 & 0.80 & 1.21 \\
\hline 813 & 0.205 & 91.3 & 39.4 & 22 & Sbc & 4.86 & -21.86 & 1.97 & 3.55 & 1.41 & 0.50 & 0.66 & - & 0.75 & 0.82 \\
\hline 814 & 0.178 & 114.1 & 36.4 & 14 & E5 & 28.71 & -22.73 & 3.29 & 2.86 & 0.59 & 0.26 & 0.31 & - & 0.40 & 0.30 \\
\hline 815 & 0.306 & 133.5 & 65.1 & 19 & E4 & 10.02 & -22.08 & 3.03 & 3.14 & 0.04 & 0.04 & 0.06 & - & 0.06 & 0.06 \\
\hline 816 & 0.273 & 157.1 & 45.4 & 9 & E5 & 10.14 & -21.88 & 3.28 & 2.92 & 0.10 & 0.27 & 0.30 & 0.34 & 0.35 & 0.32 \\
\hline 817 & 0.626 & 28.9 & 72.5 & 22 & Scd & 0.70 & -20.26 & 2.14 & 2.01 & 0.55 & 0.61 & 0.68 & - & 0.69 & 0.91 \\
\hline 818 & 0.775 & 53.6 & 80.5 & 18 & $\mathrm{Sab}$ & 3.32 & -20.55 & 2.49 & 2.79 & 1.65 & 0.60 & 0.73 & - & 0.73 & 0.99 \\
\hline 820 & 0.382 & 0.1 & 55.1 & 27 & Sbc & 3.37 & -21.30 & 1.96 & 3.21 & 0.56 & 0.59 & 0.68 & - & 0.71 & 0.92 \\
\hline 821 & 0.566 & 102.0 & 68.9 & 28 & $\mathrm{Sb}$ & 8.59 & -22.23 & 2.22 & 2.93 & 3.26 & 0.67 & 0.78 & - & 0.79 & 1.11 \\
\hline 822 & 0.380 & 133.6 & 54.1 & 19 & SOa & 9.93 & -22.32 & 2.55 & 2.79 & 1.76 & 0.29 & 0.42 & - & 0.45 & 0.46 \\
\hline 823 & 0.443 & 156.6 & 59.4 & 20 & Sbc & 1.38 & -20.55 & 1.89 & 2.47 & 0.47 & & 0.83 & - & 0.85 & 1.60 \\
\hline 824 & 0.570 & 157.3 & 68.9 & 20 & $\mathrm{Sb}$ & 4.67 & -21.46 & 2.12 & 2.35 & 1.46 & 0.64 & 0.82 & - & 0.83 & 1.43 \\
\hline 825 & 0.776 & 162.2 & 80.5 & 20 & $\mathrm{Sbc}$ & 1.21 & -20.47 & 2.54 & 2.10 & - & 0.75 & 0.82 & - & 0.82 & 1.31 \\
\hline 826 & 0.587 & 128.2 & 69.7 & 12 & SOa & 13.12 & -21.95 & 3.39 & 2.96 & 0.60 & 0.32 & 0.40 & 0.57 & 0.41 & 0.39 \\
\hline 827 & 0.826 & 179.9 & 82.8 & 18 & $\mathrm{Sc}$ & 0.29 & -19.11 & 2.36 & 2.02 & 0.19 & 0.46 & 0.60 & 0.75 & 0.60 & 0.70 \\
\hline 828 & 0.761 & 140.8 & 79.8 & 19 & $\mathrm{Sc}$ & 0.77 & -20.43 & 2.41 & 1.76 & 2.23 & 0.39 & 0.48 & 0.56 & 0.49 & 0.51 \\
\hline 829 & 0.043 & 4.8 & 17.7 & 21 & E1 & 24.21 & -22.86 & 3.19 & 2.77 & 0.06 & 0.09 & 0.10 & - & 0.23 & 0.10 \\
\hline 830 & 0.716 & 63.9 & 77.8 & 17 & $\mathrm{Sb}$ & 10.79 & -22.17 & 2.40 & 2.68 & 3.04 & 0.73 & 0.82 & - & 0.82 & 1.41 \\
\hline 831 & 0.644 & 125.0 & 73.7 & 14 & Sbc & 1.63 & -21.04 & 2.48 & 2.07 & 1.71 & 0.68 & 0.73 & 0.79 & 0.74 & 1.06 \\
\hline 832 & 0.242 & 73.3 & 42.6 & 15 & E5 & 42.56 & -23.48 & 3.36 & 2.75 & 1.12 & 0.23 & 0.26 & - & 0.31 & 0.25 \\
\hline 834 & 0.791 & 108.2 & 81.3 & 12 & $\mathrm{Sb}$ & 10.21 & -21.85 & 2.78 & 2.86 & 1.64 & 0.40 & 0.59 & 0.66 & 0.59 & 0.68 \\
\hline 835 & 0.447 & 58.7 & 59.9 & 11 & E7 & 26.67 & -22.65 & 2.91 & 2.88 & 0.09 & 0.37 & 0.51 & 0.65 & 0.53 & 0.51 \\
\hline 837 & 0.729 & 92.8 & 78.7 & 11 & $\mathrm{Sb}$ & 1.87 & -20.73 & 2.56 & 1.97 & 4.59 & 0.67 & 0.80 & 0.86 & 0.80 & 1.21 \\
\hline 838 & 0.696 & 128.4 & 76.8 & 11 & $\mathrm{Sa}$ & 6.56 & -21.19 & 3.11 & 2.67 & 0.12 & 0.45 & 0.58 & 0.78 & 0.58 & 0.60 \\
\hline 840 & 0.371 & 144.5 & 71.4 & 38 & E6 & 53.09 & -23.85 & 3.15 & 2.92 & 0.21 & 0.10 & 0.09 & - & 0.10 & 0.12 \\
\hline 841 & 0.769 & 110.2 & 80.3 & 26 & $\mathrm{Sc}$ & 0.73 & -20.00 & 2.02 & 2.24 & 0.71 & 0.44 & 0.67 & - & 0.67 & 0.81 \\
\hline 842 & 0.228 & 49.6 & 41.3 & 20 & $\mathrm{Sb}$ & 3.71 & -21.24 & 2.44 & 2.77 & 2.09 & 0.47 & 0.60 & - & 0.68 & 0.66 \\
\hline 843 & 0.782 & 22.3 & 80.5 & 23 & Scd & 0.20 & -19.32 & - & 2.46 & 0.50 & 0.25 & 0.33 & - & 0.33 & 0.22 \\
\hline 844 & 0.416 & 126.4 & 57.3 & 11 & SOa & 8.30 & -21.49 & 3.40 & 3.11 & 0.06 & 0.28 & 0.41 & 0.63 & 0.43 & 0.37 \\
\hline 845 & 0.371 & 103.8 & 71.2 & 22 & E7 & 32.14 & -23.39 & 3.28 & 2.99 & 0.16 & 0.09 & 0.13 & - & 0.14 & 0.14 \\
\hline 846 & 0.354 & 110.8 & 69.8 & 24 & E5 & 19.95 & -22.70 & 2.80 & 2.78 & 0.55 & 0.06 & 0.06 & - & 0.07 & 0.07 \\
\hline 847 & 0.652 & 147.2 & 74.4 & 20 & $\mathrm{Sb}$ & 9.68 & -22.12 & 2.83 & 2.73 & - & 0.65 & 0.75 & - & 0.75 & 1.00 \\
\hline 848 & 0.720 & 69.9 & 78.1 & 23 & $\mathrm{Sb}$ & 3.64 & -20.99 & 2.33 & 2.78 & 0.44 & 0.58 & 0.78 & - & 0.79 & 1.08 \\
\hline 849 & 0.395 & 108.5 & 55.8 & 24 & $\mathrm{Sbc}$ & 10.42 & -22.76 & 2.04 & 2.22 & 3.51 & 0.82 & 0.88 & - & 0.89 & 1.96 \\
\hline 850 & 0.466 & 173.4 & 61.1 & 12 & $\mathrm{Sab}$ & 16.26 & -22.46 & 3.34 & 2.66 & 0.59 & 0.29 & 0.35 & 0.42 & 0.36 & 0.32 \\
\hline 851 & 0.375 & 15.1 & 71.5 & 28 & E5 & 49.09 & -23.48 & 2.81 & 3.27 & 0.47 & 0.09 & 0.09 & - & 0.09 & 0.10 \\
\hline 852 & 0.416 & 59.0 & 57.6 & 20 & Scd & 0.28 & -19.56 & 2.41 & 1.80 & 0.26 & 0.19 & 0.24 & - & 0.26 & 0.28 \\
\hline
\end{tabular}


Table B.1. continued.

\begin{tabular}{|c|c|c|c|c|c|c|c|c|c|c|c|c|c|c|c|}
\hline ID & $\epsilon$ & $\begin{array}{c}\text { PA } \\
(\mathrm{deg})\end{array}$ & $\begin{array}{l}\text { Incl. } \\
\text { (deg) }\end{array}$ & $\begin{array}{c}R_{\text {eff }} \\
(\operatorname{arcsec})\end{array}$ & Type & $\begin{array}{c}M_{*} \\
\left(10^{10} M_{\odot}\right)\end{array}$ & $\begin{array}{c}M_{r} \\
\text { (mag) }\end{array}$ & $C_{90 / 50}$ & $\begin{array}{c}u-r \\
(\mathrm{mag})\end{array}$ & $\begin{array}{c}\mathrm{SFR} \\
\left(M_{\odot} \mathrm{yr}^{-1}\right)\end{array}$ & $\lambda_{0.5 \mathrm{Re}}$ & $\lambda_{\operatorname{Re}}$ & $\lambda_{2 \operatorname{Re}}$ & $\lambda_{\mathrm{Re}, 90^{\circ}}$ & $(V / \sigma)_{\mathrm{e}}$ \\
\hline 854 & 0.662 & 91.6 & 75.2 & 12 & $\mathrm{Sb}$ & 7.48 & -21.92 & 2.69 & 2.48 & 1.22 & 0.55 & 0.66 & 0.82 & 0.67 & 0.79 \\
\hline 856 & 0.358 & 89.1 & 52.7 & 17 & $\mathrm{Sb}$ & 2.00 & -21.18 & 2.19 & 2.32 & 1.49 & 0.69 & 0.76 & - & 0.79 & 1.18 \\
\hline 857 & 0.640 & 42.6 & 73.6 & 14 & $\mathrm{Sbc}$ & 7.87 & -21.87 & 2.10 & 2.60 & 3.51 & 0.70 & 0.81 & - & 0.81 & 1.29 \\
\hline 858 & 0.555 & 173.1 & 67.8 & 15 & $\mathrm{SOa}$ & 40.46 & -22.92 & 3.47 & 2.95 & 0.64 & 0.50 & 0.60 & - & 0.61 & 0.64 \\
\hline 859 & 0.355 & 65.3 & 70.0 & 34 & E4 & 12.08 & -22.42 & 3.00 & 3.23 & 0.06 & 0.09 & 0.08 & - & 0.09 & 0.09 \\
\hline 860 & 0.569 & 34.6 & 68.7 & 8 & So & 5.64 & -21.37 & 3.25 & 2.64 & 0.05 & 0.34 & 0.39 & 0.55 & 0.40 & 0.37 \\
\hline 861 & 0.780 & 54.1 & 80.6 & 24 & Sbc & 2.66 & -21.22 & 2.34 & 2.57 & 0.83 & 0.71 & 0.85 & - & 0.85 & 1.40 \\
\hline 862 & 0.632 & 34.2 & 73.2 & 23 & $\mathrm{Sc}$ & 10.33 & -22.57 & 2.05 & 2.40 & 7.00 & 0.67 & 0.82 & - & 0.82 & 1.25 \\
\hline 863 & 0.513 & 112.3 & 64.6 & 13 & Sab & 10.89 & -22.09 & 2.66 & 3.16 & 1.45 & 0.43 & 0.57 & 0.79 & 0.59 & 0.62 \\
\hline 864 & 0.349 & 12.0 & 69.2 & 19 & E3 & 15.60 & -22.73 & 3.13 & 2.85 & 0.14 & 0.04 & 0.06 & - & 0.06 & 0.07 \\
\hline 865 & 0.326 & 178.5 & 50.6 & 12 & So & 12.39 & -22.10 & 3.42 & 2.87 & 0.76 & 0.26 & 0.38 & 0.57 & 0.42 & 0.36 \\
\hline 867 & 0.716 & 119.0 & 77.9 & 9 & $\mathrm{Sab}$ & 2.42 & -20.46 & 2.94 & 2.65 & 0.10 & 0.45 & 0.59 & 0.72 & 0.59 & 0.63 \\
\hline 868 & 0.638 & 159.5 & 73.4 & 17 & $\mathrm{Sb}$ & 4.79 & -21.69 & 2.18 & 2.56 & 2.64 & 0.77 & 0.85 & - & 0.86 & 1.48 \\
\hline 869 & 0.256 & 131.1 & 43.9 & 20 & $\mathrm{Sb}$ & 8.77 & -22.39 & 2.12 & 2.91 & 2.13 & 0.54 & 0.74 & - & 0.80 & 0.95 \\
\hline 870 & 0.360 & 127.1 & 53.0 & 13 & S0 & 24.49 & -21.91 & 2.96 & 2.89 & 0.00 & 0.34 & 0.51 & 0.68 & 0.55 & 0.54 \\
\hline 871 & 0.575 & 126.8 & 69.1 & 18 & $\mathrm{Sb}$ & 10.79 & -22.15 & 2.41 & 2.85 & 1.21 & 0.73 & 0.83 & - & 0.83 & 1.31 \\
\hline 872 & 0.217 & 63.5 & 40.4 & 19 & Sab & 3.70 & -21.38 & 2.12 & 3.16 & 0.29 & 0.45 & 0.62 & - & 0.70 & 0.71 \\
\hline 873 & 0.456 & 57.3 & 60.2 & 16 & $\mathrm{Sb}$ & 12.39 & -22.56 & 2.51 & 3.76 & 2.04 & 0.49 & 0.57 & - & 0.59 & 0.68 \\
\hline 874 & 0.325 & 39.9 & 50.1 & 13 & SOa & 33.65 & -22.73 & 3.27 & 3.21 & 0.51 & 0.32 & 0.38 & 0.51 & 0.42 & 0.39 \\
\hline 876 & 0.450 & 107.7 & 60.1 & 18 & Sbc & 6.18 & -21.83 & 2.04 & 3.07 & 1.82 & 0.71 & 0.83 & - & 0.85 & 1.49 \\
\hline 877 & 0.610 & 37.1 & 71.6 & 17 & Sab & 6.95 & -21.84 & - & 3.31 & 2.50 & 0.50 & 0.60 & 0.65 & 0.60 & 0.68 \\
\hline 878 & 0.779 & 32.1 & 80.6 & 24 & Scd & 0.11 & -18.77 & 2.29 & 1.72 & 0.35 & 0.47 & 0.57 & - & 0.57 & 0.69 \\
\hline 881 & 0.300 & 18.5 & 47.8 & 17 & E3 & 27.86 & -23.05 & 3.27 & 2.93 & 0.15 & 0.35 & 0.38 & - & 0.43 & 0.39 \\
\hline 885 & 0.739 & 47.4 & 79.1 & 15 & $\mathrm{Sc}$ & 0.10 & -18.41 & 2.67 & 1.67 & 0.17 & 0.18 & 0.41 & 0.51 & 0.41 & 0.44 \\
\hline 886 & 0.473 & 9.9 & 61.7 & 12 & $\mathrm{Sa}$ & 11.72 & -22.45 & 3.06 & 2.90 & 2.76 & 0.51 & 0.64 & 0.76 & 0.66 & 0.72 \\
\hline 887 & 0.324 & 14.4 & 49.8 & 15 & Sbc & 8.53 & -22.48 & 2.39 & 2.5 & 5.20 & 0.60 & 0.76 & 0.82 & 0.8 & 1.12 \\
\hline 888 & 0.107 & 0.3 & 35.4 & 36 & $\mathrm{E} 1$ & 23.39 & -23.47 & 3.05 & 3.50 & 0.23 & 0.06 & 0.06 & - & 0.09 & 0.06 \\
\hline 889 & 0.320 & 65.0 & 49.5 & 12 & $\mathrm{Sab}$ & 7.62 & -22.04 & 3.11 & 2.51 & 4.37 & 0.51 & 0.61 & 0.62 & 0.65 & 0.75 \\
\hline 890 & 0.561 & 159.2 & 68.6 & 12 & $\mathrm{Sb}$ & 4.68 & -21.56 & 2.83 & 2.75 & 0.81 & 0.46 & 0.66 & 0.78 & 0.67 & 0.78 \\
\hline 892 & 0.772 & 70.8 & 80.4 & 23 & $\mathrm{Sb}$ & 2.32 & -20.96 & 3.11 & 3.23 & 2.05 & 0.68 & 0.75 & - & & 0.98 \\
\hline 893 & 0.153 & 41.9 & 43.4 & 27 & $\mathrm{E} 2$ & 82.04 & -23.50 & 2.86 & 3.42 & 0.26 & & 0.12 & - & & 0.12 \\
\hline 894 & 0.678 & 143.1 & 76.0 & 17 & $\mathrm{Sa}$ & 14.19 & -22.08 & 2.72 & 3.44 & 0.32 & 0.51 & 0.65 & - & 0.65 & 0.72 \\
\hline 895 & 0.794 & 118.0 & 81.4 & 27 & Scd & 0.11 & -18.88 & 2.45 & 2.01 & 0.20 & 0.43 & 0.55 & - & 0.55 & 0.60 \\
\hline 896 & 0.623 & 25.7 & 72.8 & 13 & Sbc & 5.60 & -21.86 & 2.69 & 2.53 & 4.35 & 0.55 & 0.64 & 0.77 & 0.64 & 0.74 \\
\hline 898 & 0.472 & 160.6 & 61.8 & 20 & Sbc & 3.17 & -22.07 & 2.28 & 2.41 & 7.00 & 0.76 & 0.78 & - & 0.8 & 1.33 \\
\hline 900 & 0.095 & 154.0 & 33.3 & 24 & $\mathrm{E} 4$ & 27.04 & -22.89 & 2.89 & 3.13 & 0.33 & 0.05 & 0.08 & - & 0.13 & 0.08 \\
\hline 901 & 0.603 & 16.1 & 71.1 & 20 & Sbc & 3.97 & -21.75 & 2.13 & 2.81 & 2.60 & 0.55 & 0.63 & - & 0.63 & 0.78 \\
\hline 902 & 0.468 & 149.6 & 61.4 & 9 & $\mathrm{Sa}$ & 9.18 & -21.54 & 3.52 & 3.13 & 0.06 & 0.23 & 0.31 & 0.46 & 0.32 & 0.29 \\
\hline 903 & 0.324 & 83.0 & 67.0 & 20 & E4 & 17.66 & -22.54 & 3.07 & 3.11 & 0.07 & 0.05 & 0.10 & - & 0.11 & 0.09 \\
\hline 904 & 0.455 & 150.2 & 60.4 & 16 & Sbc & 5.75 & -21.91 & 2.97 & 2.63 & 7.37 & 0.61 & 0.66 & - & 0.68 & 0.82 \\
\hline 905 & 0.666 & 37.5 & 75.3 & 20 & $\mathrm{Sd}$ & 0.28 & -19.67 & 2.38 & 2.02 & - & 0.49 & 0.49 & - & 0.49 & 0.59 \\
\hline 906 & 0.545 & 25.1 & 67.1 & 17 & $\mathrm{Sc}$ & 1.89 & -20.61 & - & 2.71 & 1.68 & 0.57 & 0.68 & - & 0.69 & 0.86 \\
\hline 907 & 0.732 & 18.5 & 78.5 & 20 & $\mathrm{Sbc}$ & 1.24 & -20.00 & 2.26 & 2.77 & 0.49 & 0.67 & 0.82 & - & 0.82 & 1.31 \\
\hline 908 & 0.550 & 134.9 & 67.2 & 11 & So & 7.93 & -21.32 & 3.52 & 2.83 & 0.01 & 0.38 & 0.54 & - & 0.56 & 0.60 \\
\hline 909 & 0.701 & 157.9 & 76.9 & 21 & $\mathrm{Sc}$ & 1.09 & -20.56 & 2.30 & 2.67 & 1.96 & 0.68 & 0.77 & - & 0.78 & 1.31 \\
\hline 910 & 0.644 & 23.9 & 73.8 & 17 & $\mathrm{Sb}$ & 1.80 & -19.45 & 2.79 & 3.18 & 0.06 & 0.67 & 0.91 & - & 0.91 & 4.78 \\
\hline 911 & 0.174 & 50.2 & 46.7 & 35 & E3 & 8.79 & -22.69 & 3.25 & 3.10 & 0.10 & 0.14 & 0.13 & - & 0.16 & 0.15 \\
\hline 912 & 0.300 & 7.9 & 47.9 & 10 & So & 9.57 & -21.47 & 3.41 & 2.83 & 0.03 & 0.19 & 0.27 & 0.44 & 0.31 & 0.25 \\
\hline 913 & 0.044 & 10.9 & 17.8 & 14 & $\mathrm{Sa}$ & 1.33 & -20.26 & 2.61 & 2.43 & 1.59 & 0.43 & 0.49 & 0.56 & 0.80 & 0.59 \\
\hline 914 & 0.615 & 76.0 & 72.1 & 17 & $\mathrm{Sb}$ & 3.38 & -21.10 & 2.56 & 2.72 & 0.88 & 0.63 & 0.77 & - & 0.78 & 1.12 \\
\hline 915 & 0.182 & -11.0 & 36.7 & 12 & $\mathrm{Sb}$ & 3.16 & -21.58 & 2.73 & 2.28 & 2.68 & 0.49 & 0.57 & 0.63 & 0.68 & 0.66 \\
\hline 916 & 0.372 & 133.9 & 53.9 & 11 & So & 9.04 & -21.76 & 3.22 & 2.90 & 0.11 & 0.34 & 0.47 & 0.59 & 0.50 & 0.46 \\
\hline 917 & 0.477 & 138.5 & 62.2 & 14 & So & 10.45 & -21.74 & 3.16 & 3.07 & 0.09 & 0.42 & 0.49 & 0.64 & 0.51 & 0.51 \\
\hline 919 & 0.656 & 22.2 & 74.5 & 9 & S0 & 9.68 & -21.69 & 3.06 & 2.90 & 0.43 & 0.39 & 0.58 & 0.75 & 0.58 & 0.58 \\
\hline 920 & 0.211 & 171.9 & 39.9 & 28 & Sbc & 1.64 & -21.34 & 1.97 & 2.92 & - & 0.65 & 0.66 & - & 0.75 & 0.98 \\
\hline 923 & 0.546 & 92.1 & 67.3 & 15 & E7 & 11.30 & -22.02 & 3.29 & 2.86 & 0.11 & 0.35 & 0.43 & 0.50 & 0.44 & 0.41 \\
\hline 924 & 0.194 & 31.6 & 38.0 & 21 & $\mathrm{Sb}$ & 2.45 & -21.04 & 2.73 & 2.57 & 0.49 & 0.48 & 0.57 & - & 0.67 & 0.64 \\
\hline 925 & 0.273 & 148.8 & 45.6 & 21 & $\mathrm{Sab}$ & 17.58 & -22.05 & 2.63 & 3.25 & 0.84 & 0.40 & 0.43 & - & 0.50 & 0.48 \\
\hline
\end{tabular}


Table B.1. continued.

\begin{tabular}{cccccccccccccccc}
\hline \hline ID & $\epsilon$ & $\begin{array}{c}\text { PA } \\
(\mathrm{deg})\end{array}$ & $\begin{array}{c}\text { Incl. } \\
(\mathrm{deg})\end{array}$ & $\begin{array}{c}R_{\mathrm{eff}} \\
(\mathrm{arcsec})\end{array}$ & $\begin{array}{c}\text { Type } \\
\left(10^{10} M_{\odot}\right)\end{array}$ & $\begin{array}{c}M_{r} \\
(\mathrm{mag})\end{array}$ & $C_{90 / 50}$ & $\begin{array}{c}u-r \\
(\mathrm{mag})\end{array}$ & $\begin{array}{c}\mathrm{SFR} \\
\left(M_{\odot} \mathrm{yr}^{-1}\right)\end{array}$ & $\lambda_{0.5 \mathrm{Re}}$ & $\lambda_{\mathrm{Re}}$ & $\lambda_{2 \mathrm{Re}}$ & $\lambda_{\mathrm{Re}, 90^{\circ}}$ & $(V / \sigma)_{\mathrm{e}}$ \\
\hline 926 & 0.820 & 75.5 & 82.4 & 17 & $\mathrm{Sc}$ & 0.76 & -19.77 & 2.07 & 1.93 & 0.64 & 0.60 & 0.79 & - & 0.79 & 1.39 \\
927 & 0.587 & 34.7 & 70.2 & 14 & $\mathrm{Sb}$ & 12.00 & -22.23 & 2.96 & 2.81 & 15.20 & 0.39 & 0.42 & 0.61 & 0.43 & 0.49 \\
929 & 0.610 & 56.0 & 71.5 & 20 & $\mathrm{Sbc}$ & 5.43 & -22.01 & 2.00 & 2.61 & 3.79 & 0.69 & 0.84 & - & 0.84 & 1.40 \\
930 & 0.502 & 140.4 & 63.6 & 16 & $\mathrm{Sc}$ & 0.66 & -20.63 & 2.37 & 1.97 & 1.05 & 0.57 & 0.66 & - & 0.68 & 0.85 \\
932 & 0.535 & 120.2 & 66.3 & 15 & $\mathrm{Sa}$ & 28.51 & -22.59 & 3.04 & 2.92 & 0.48 & 0.48 & 0.55 & - & 0.56 & 0.60 \\
933 & 0.715 & 104.3 & 77.6 & 11 & $\mathrm{Sab}$ & 4.18 & -21.17 & 2.56 & 2.71 & 2.46 & 0.50 & 0.58 & - & 0.59 & 0.69 \\
934 & 0.722 & 35.4 & 77.8 & 19 & $\mathrm{Sbc}$ & 0.56 & -19.49 & 2.47 & 2.37 & 0.40 & 0.43 & 0.48 & - & 0.49 & 0.53 \\
935 & 0.611 & 110.0 & 71.7 & 27 & $\mathrm{Sc}$ & 1.13 & -20.69 & 2.39 & 2.78 & 0.97 & 0.34 & 0.50 & - & 0.51 & 0.51 \\
937 & 0.607 & 44.8 & 71.1 & 32 & $\mathrm{Ir}$ & 0.19 & -19.56 & 2.44 & 1.51 & 0.40 & 0.18 & 0.19 & - & 0.19 & 0.22 \\
2999 & 0.154 & 61.6 & 33.8 & 13 & $\mathrm{Sbc}$ & 3.48 & -21.56 & 2.36 & 2.65 & 1.86 & 0.44 & 0.62 & 0.74 & 0.74 & 0.70 \\
4034 & 0.438 & 43.4 & 59.1 & 15 & $\mathrm{~S} 0$ & 7.18 & -22.09 & 2.01 & 2.83 & 1.90 & 0.26 & 0.34 & - & 0.36 & 0.36 \\
\hline
\end{tabular}

